\title{
New approach to LHC optics commissioning for the nonlinear era
}

\author{
E. H. Maclean ${ }^{*}$ \\ CERN, Geneva CH-1211, Switzerland and University of Malta, Msida MSD 2080, Malta \\ R. Tomás, F. S. Carlier, M. S. Camillocci, J. W. Dilly, J. Coello de Portugal, E. Fol, \\ K. Fuchsberger, A. Garcia-Tabares Valdivieso, M. Giovannozzi, M. Hofer, \\ L. Malina, T. H. B. Persson, P. K. Skowronski, and A. Wegscheider \\ CERN, Geneva CH-1211, Switzerland
}

(Received 16 October 2018; published 21 June 2019)

\begin{abstract}
In 2017, optics commissioning strategy for low- $\beta^{*}$ operation of the CERN Large Hadron Collider (LHC) underwent a major revision. This was prompted by a need to extend the scope of beam-based commissioning at high energy, beyond the exclusively linear realm considered previously, and into the nonlinear regime. It also stemmed from a recognition that, due to operation with crossing angles in the experimental insertions, the linear and nonlinear optics quality were intrinsically linked through potentially significant feed-down at these locations. Following the usual linear optics commissioning therefore, corrections for (normal and skew) sextupole and (normal and skew) octupole errors in the high-luminosity insertions were implemented. For the first time, the LHC now operates at top energy with beam-based corrections for nonlinear dynamics, and for the effect of the crossing scheme on beta-beating and dispersion. The new commissioning procedure has improved the control of various linear and nonlinear characteristics of the LHC, yielding clear operational benefits.
\end{abstract}

DOI: 10.1103/PhysRevAccelBeams.22.061004

\section{INTRODUCTION}

Control of linear optics is a key operational concern at the CERN Large Hadron Collider (LHC). Machine protection, as well as an equitable distribution of delivered luminosity to the various high energy physics (HEP) apparatus, require tight constraints on optics quality. During the 2012-2016 period, development of new tools and methodology for LHC beam commissioning [1-4] allowed an unprecedented degree of control for hadron colliders to be achieved over linear optics [5-7]. In 2017 however, a major revision to the commissioning strategy for low- $\beta^{*}$ optics was introduced. The objective of this change was to extend LHC optics commissioning into the nonlinear regime.

The impact of nonlinear magnetic errors in low- $\beta^{*}$ insertions (IRs) increase substantially as $\beta^{*}$ is reduced. Such IR-nonlinearities were a concern during design and construction of the Tevatron [8], RHIC [9], and LHC [10], with beam-based optimization of lifetime via nonlinear correctors also yielding operational benefits at the RHIC

\footnotetext{
*ewen.hamish.maclean@cern.ch
}

Published by the American Physical Society under the terms of the Creative Commons Attribution 4.0 International license. Further distribution of this work must maintain attribution to the author(s) and the published article's title, journal citation, and DOI. collider [11,12]. In the High-Luminosity LHC (HL-LHC) [13] compensation of nonlinear errors in experimental IRs is expected to be an operational necessity [14-16], with similar limitations expected for SuperKEKB [17] and the Future Circular Collider (FCC) lattice [18-20].

Prior to 2017 the LHC operated without any correction for nonlinear errors in its low- $\beta^{*}$ experimental insertions. Measurement of the uncompensated amplitude detuning however, demonstrated that for operational $\beta^{*}$ the tunespread generated by normal octupole errors in the IRs was comparable to that purposefully introduced by ("Landau") octupoles in the arcs to provide Landau damping [21]. This leads to a radical distortion of tune footprint through the operational cycle, as illustrated in Fig. 1, which shows that for $\beta^{*} \lesssim 0.4 \mathrm{~m}$ the footprint generated by the combination of Landau octupoles and IR-tunespread (red) differs substantially from that desired (gray). Such distortions influence the understanding and mitigation of instabilities [21] and clear effects of IR-octupoles were observed on the instability threshold at $\beta^{*}=0.4 \mathrm{~m}$ [22]. In 2017 the LHC initially operated at $\beta^{*}=0.4 \mathrm{~m}$, then $0.3 \mathrm{~m}$. In 2018 it operated down to $\beta^{*}=0.25 \mathrm{~m}$. There is thus a strong motivation to compensate IR-octupole errors in the LHC.

Linear coupling can also cause substantial changes to detuning coefficients [23-26], and plays a significant role in the generation of amplitude-dependent closest tune approach [23,27-31]. Such coupling-induced distortions of tune-footprint are detrimental to Landau damping of 

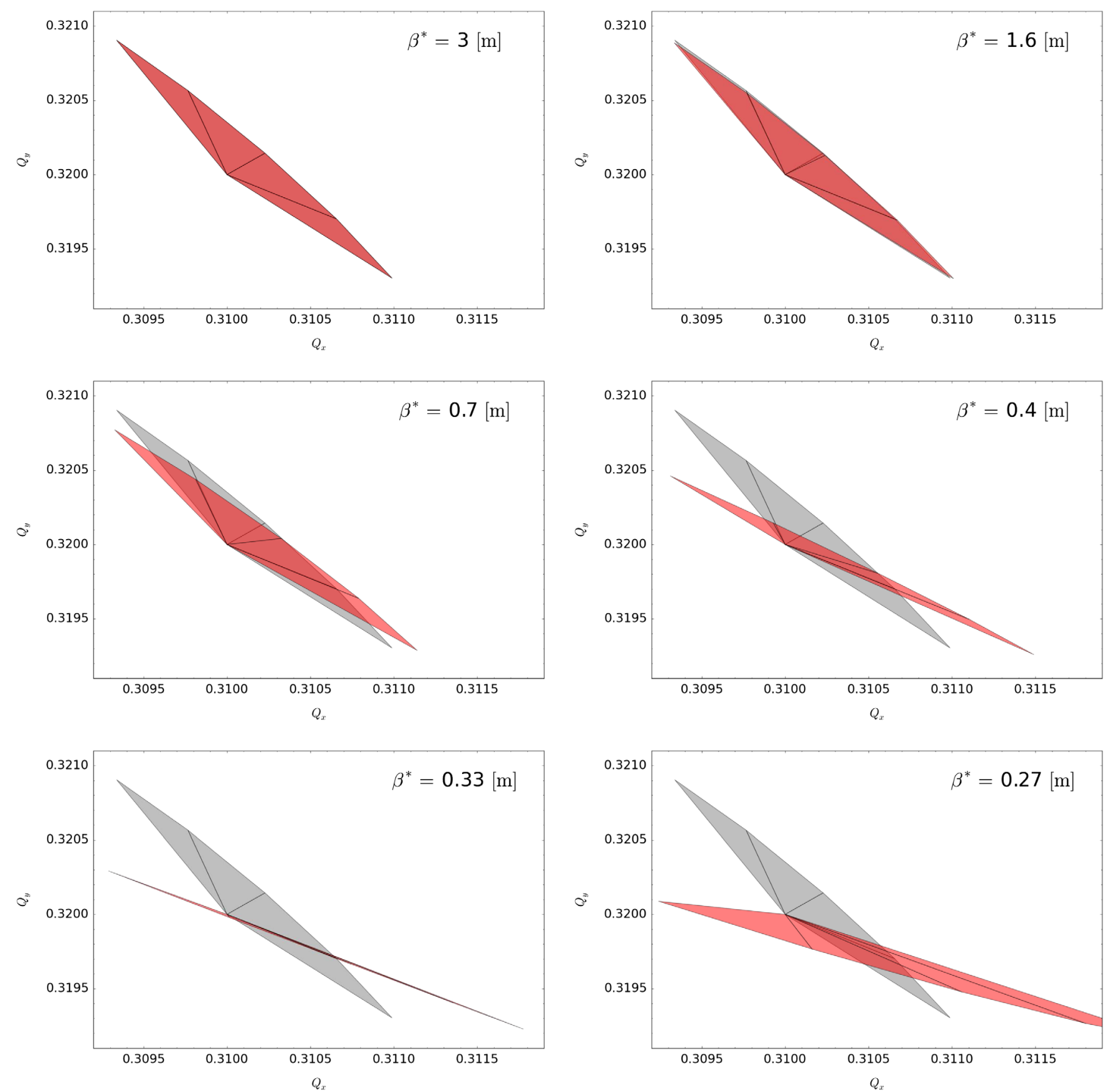

FIG. 1. Distortion of tune footprint through the $\beta^{*}$ squeeze. Displayed footprints are defined by first-order detuning coefficients obtained via simulation. Simulations consist of an effective model of normal octupole errors in IR1 (ATLAS) and IR5 (CMS) which reproduces the observed detuning, together with Landau octupoles powered as per operation for luminosity production in 2016. Grey regions show the desired footprint, expected in the absence of the IR contribution. Red regions show the inferred footprint if IR-octupole errors are uncompensated.

instabilities, as demonstrated in the LHC $[32,33]$. During luminosity production the LHC operates with crossing angle orbit bumps in the experimental insertions. As a consequence of these bumps, IR-nonlinear errors can generate substantial feed-down to linear coupling. While such feed-down could in principle be corrected globally for a specific optics and crossing scheme, introduction of crossing angle leveling and $\beta^{*}$-leveling into regular LHC operation, as well as routine manipulations of IR-orbit bumps during machine studies, gave significant motivation for compensation of the relevant multipole errors.

Feed-down arising from crossing angles in the experimental IRs will not only perturb linear coupling, but also linear optics. Improvements to linear optics quality have a positive influence on a broad range a machine parameters [6], and any potential source of luminosity imbalance 
between the ATLAS and CMS experiments is of great concern. In all previous years, commissioning of linear optics in the LHC has been performed at flat orbit (a closed orbit without any orbit bumps in the experimental IRs), and thus neglected any optics perturbation from feed-down in the low- $\beta^{*}$ insertions. In 2017, to ensure the smallest possible $\beta^{*}$-imbalance between the HEP experiments, not only was it decided to attempt direct correction of the relevant nonlinear sources in the IRs, but also to introduce, for the first time, a linear reoptimization of the optics at the operational crossing scheme after all higher-order corrections were applied.

The LHC now operates with dedicated corrections for nonlinear errors in its low- $\beta^{*}$ insertions, and for the impact of the operational crossing scheme on the linear optics. The revised commissioning procedure represents a significant evolution of the previous strategy employed at the LHC. This paper reports the results of the first combined linear and nonlinear optics commissioning of the LHC. Section II describes an initial linear optics commissioning at flat orbit. Section III describes the methodology and results of the first nonlinear commissioning of the LHC. Finally Sec. IV will report on the first linear optics commissioning of the LHC at the operational crossing scheme, and present results for the final optics quality. An accompanying note as also been produced, which provides additional detail [34].

\section{LINEAR OPTICS COMMISSIONING WITH A FLAT ORBIT}

In the absence of any beam-based linear optics corrections the $\beta$-beat $(\Delta \beta / \beta)$ in the LHC at end-of-squeeze is of the order of $\sim 100 \%[6,7]$. Correction of nonlinear optics relies on an accurate response of the various corrector circuits, and is thus sensitive to linear optics errors. To establish a baseline linear optics in good agreement with the nominal model, and therefore facilitate nonlinear correction, an initial round of linear optics corrections were performed with a flat closed orbit. This phase of the commissioning replicated the exclusively linear commissioning strategy employed in the LHC prior to 2017. Detailed descriptions of the methods used in LHC linear optics commissioning may be found in $[1-7,35,36]$ and an overview of these methods within the broader context of optics measurement techniques may be found in [37]. It should also be noted that in 2017 the operational LHC optics transitioned from its nominal configuration to the achromatic telescopic squeeze (ATS) scheme $[38,39]$.

Local corrections for large quadrupole errors in the insertions were retained from earlier studies [40] which reduced the $\Delta \beta / \beta$ to $\sim 10 \%$. Figure 2 then shows an example of the $\beta$-beat obtained at flat orbit before and after further global optimization for $\beta^{*}$, inter-BPM phase advances, and normalized dispersion $\left(D_{x} / \sqrt{\beta}\right.$ [35]) via all available quadrupole circuits. Table I compares final linear
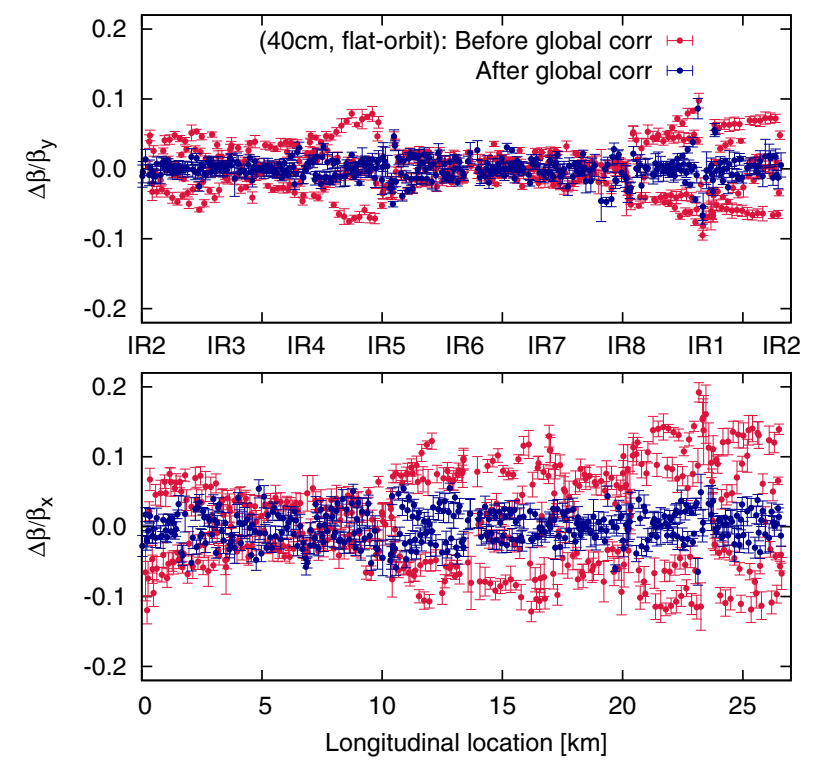

FIG. 2. $\beta$-beat in LHC Beam 1 before and after application of global optics correction at flat orbit in 2017.

optics quality, obtained at flat orbit and $\beta^{*}=0.4 \mathrm{~m}$ in 2017 and 2016 [7].

A minor deterioration (at the level of $\sim 1 \%$ ) can be seen in the postcorrection RMS $\beta$-beat of the horizontal plane. Peak values in 2017 also appear slightly worse than 2016, but are dominated by a minority of outlying BPMs. Nonetheless the linear optics quality obtained at flat orbit is comparable to that obtained in previous years [5-7], and establishes an excellent baseline optics quality upon which to implement correction for the nonlinear errors in the experimental IRs. Further details regarding the linear optics measurements are provided in the accompanying note [34].

Linear coupling in the LHC is characterized by the $f_{1001}$ resonance driving term (RDT) related to the $\left(Q_{x}-Q_{y}\right)$ resonance [41-43], and is corrected throughout the LHC cycle $[7,44]$. Given the growing appreciation of the

TABLE I. Comparison of linear optics quality obtained with flat orbit for the 2017 ATS optics at $\beta^{*}=0.4 \mathrm{~m}$, to that obtained with flat orbit in 2016 for the nominal $0.4 \mathrm{~m}$ optics. Values for 2016 were taken from [7].

\begin{tabular}{lccccc}
\hline \hline & \multicolumn{2}{c}{2017 ATS } & & \multicolumn{2}{c}{2016 Nominal } \\
\cline { 2 - 3 } & Beam 1 & Beam 2 & & Beam 1 & Beam 2 \\
\hline$\left.\beta_{x}\right|_{\text {RMS }}[\%]$ & 2.3 & 2.6 & & 1.4 & 1.4 \\
$\left.\beta_{y}\right|_{\text {RMS }}[\%]$ & 1.5 & 1.5 & & 1.8 & 1.4 \\
$\left.\beta_{x}\right|_{\text {peak }}[\%]$ & 5.5 & 10.9 & & 7.7 & 4.5 \\
$\left.\beta_{y}\right|_{\text {peak }}[\%]$ & 8.6 & 7.6 & & 5.8 & 4.9 \\
$\frac{\Delta D_{x}}{\left.\sqrt{\beta_{x}}\right|_{\text {RMS }}\left[10^{-2} \mathrm{~m}^{-\frac{1}{2}}\right]}$ & 0.45 & 0.58 & & 0.52 & 0.62 \\
$\left|\frac{\Delta D_{x}}{\sqrt{\beta_{x}}}\right|_{\text {peak }}\left[10^{-2} \mathrm{~m}^{-\frac{1}{2}}\right]$ & 1.2 & 4.3 & & 1.9 & 1.8 \\
\hline \hline
\end{tabular}


importance coupling holds to LHC operation [26,32,33] however, commissioning strategy was extended to include compensation of chromatic coupling. Chromatic coupling refers to a momentum dependent linear coupling. A firstorder dependence on the relative momentum offset can be generated by skew sextupoles in regions of horizontal dispersion, and normal sextupoles in regions of vertical dispersion. At flat orbit these sources lie primarily in the LHC arcs. Chromatic coupling is measured via the change in $f_{1001}$ with $\delta p / p$. It is compensated via skew sextupole correctors located in the arcs. Measurement and correction of chromatic coupling was previously demonstrated in the LHC [45], but was never before implemented operationally.

Figure 3 (red) shows the chromatic coupling measured after application of linear optics corrections. Chromatic coupling was particularly large in Beam 2. For a particle with relative momentum offset $\frac{\delta p}{p}=10^{-4}$ (which is representative of the nominal RMS momentum spread of LHC bunches and the typical $\mathrm{rf}$ modulation used in $Q_{x, y}^{\prime}$ measurement) the observed value is approximately equivalent to $\Delta\left|C^{-}\right| \approx 0.001$ (comparable with typical onmomentum values of the linear coupling). After correction (Fig. 3, blue) the variation of $\left|C^{-}\right|$with relative momentum offset was reduced to levels of negligible significance in both beams. For the first time the LHC now operates with chromatic coupling compensated.

\section{COMMISSIONING OF THE NONLINEAR OPTICS}

Dedicated correctors (normal/skew sextupole, normal/ skew octupole, normal dodecapole) are located on the left

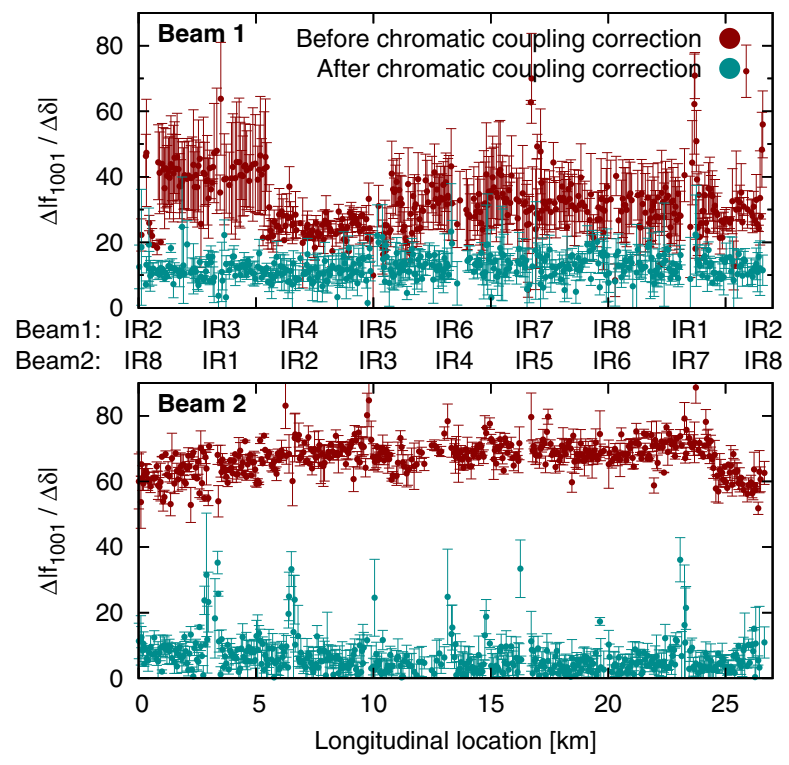

FIG. 3. Measurements of chromatic coupling before (red) and after (blue) compensation with skew sextupole correctors in the LHC arcs. Plots are shown for Beam 1 (top) and Beam 2 (bottom).

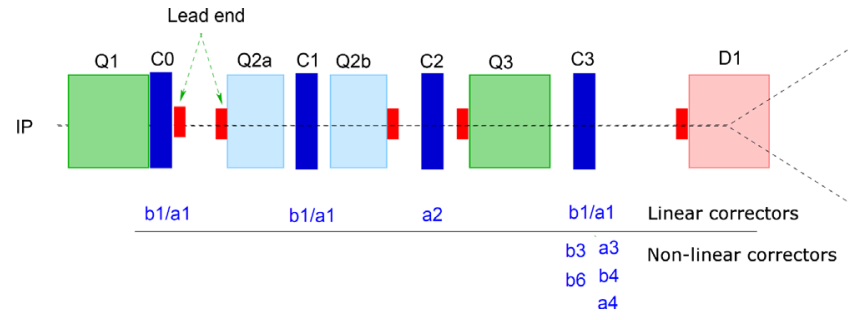

FIG. 4. Linear and nonlinear corrector layout in LHC experimental IRs [47].

and right sides of each experimental IR (location C3 in Fig. 4) for the purpose of local compensation of nonlinear errors $[10,46]$. All correctors may be powered independently, however as they are located in the region of common aperture the two LHC beams cannot be corrected independently. Since the errors also lie in the region of common aperture however, common local correction of the two beams should in general be viable. Prior to 2017 nonlinear correctors in the experimental IRs had never been used in LHC operation.

During LHC design it was assumed that nonlinear corrections in the IRs would be inferred directly from magnetic measurements performed during construction $[47,48]$. Comparison of beam-based measurements to models incorporating the magnetic measurements however, revealed significant discrepancies for several multipoles [49] which precluded straightforward application of the design strategy. Consequently greater emphasis has been placed on methods for beam-based study of the nonlinear errors in the experimental insertions at $6.5 \mathrm{TeV}$. In particular, an AC-dipole (which can repeatedly and adiabatically excite driven oscillations of the same beam [50]) is an important tool for optics study at top energy in a slowcycling machine such as the LHC. Understanding the influence of such driven oscillations on action-dependent tune shifts [24] and dynamic aperture [51,52] has enabled a beam-based approach to nonlinear optics correction at 6.5 TeV, as did refinements to methods based on feeddown to tune and linear coupling during orbit scans in the IRs $[49,53]$.

A second departure from the LHC design strategy relates to the observable quantities upon which corrections were optimized. In the design strategy is was assumed that IRnonlinear corrections would aim to compensate specifically selected RDTs [47]. This has not generally been the method employed. Measurement of high-order RDTs is challenging, and to facilitate the designed correction strategy local variations of several RDTs from any given multipole must be measurable. Further, RDTs measured by forced oscillations (labeled $f_{j k l m}^{\prime}$ as opposed to $f_{j k l m}$ ) actually correspond to a mixture of resonances of the free motion [54] creating ambiguity in the specific resonance being studied. The driven RDTs do still provide a good observable for multipole strength more generally, and work to develop the 
methodology to directly compensate high-order RDTs in the LHC is ongoing [54-56], however for the initial venture into nonlinear optics commissioning of the LHC reported here, beam-based corrections were calculated based upon measurements of amplitude detuning, and feed-down to either linear coupling or tune, with RDT measurements used to validate corrections calculated by these means.

It should be noted that given the quasilocal nature of the corrections (with correctors mounted at a single location left and right of the IR) optimal compensation of one observable, for example feed-down, may differ from ideal corrections for the corresponding RDT. This is not a fundamental problem with the adopted commissioning strategy, since in many cases the more easily observed quantities, namely detuning and feed-down, are of significantly greater operational relevance than the RDTs. For example, loss of Landau damping during crossing angle leveling due to feed-down from nonlinear errors to linear coupling can represent a more significant challenge to successful LHC operation than the corresponding high-order resonances.

Commissioning for the nonlinear errors in the LHC began with correction of normal octupole sources in the ATLAS (IR1) and CMS (IR5) insertions, described in Sec. III A, since minimizing amplitude detuning early in the commissioning period is beneficial to both the linear and lower-order nonlinear measurements and corrections. Simultaneous corrections were then applied for normal sextupole errors in the CMS insertion, and for normal sextupole, skew sextupole, and skew octupole errors in the ATLAS insertion. Sextupole correction in CMS (IR5) is discussed in Sec. III B, while the sextupole and skew octupole corrections in ATLAS (IR1) are discussed in Sec. III C.

\section{A. Normal octupole correction in the ATLAS (IR1) and CMS (IR5) insertions}

Of foremost concern for LHC operation was compensation of normal octupole errors in IR1 and IR5. First-order amplitude detuning, a linear variation of tune with particle action $\left(J_{x, y}\right)$, relates directly to the integrated octupole content of the machine, weighted by $\beta_{x, y}^{2}$ at the octupoles. At small $\beta^{*}$, with Landau octupoles depowered, amplitude detuning in the LHC is dominated by the contribution from IR1 and IR5. Figure 5 shows amplitude detuning of LHC Beam 2, measured with an ac-dipole at $\beta^{*}=0.4 \mathrm{~m}$ (flat orbit). This is compared to MAD-X/PTC [57,58] predictions in gray for models including the measured magnetic errors. Predictions are adjusted for the impact of driven oscillations with an ac-dipole [24]. Similar results were obtained for LHC Beam 1.

Cross-term detuning, $\frac{\partial Q_{x}}{\partial \epsilon_{y}}=\frac{\partial Q_{y}}{\partial \epsilon_{x}}$, (where $\epsilon_{x, y}=2 J_{x, y}$ ) was consistent with zero within measurement errors, as expected from the magnetic model. It is not shown in
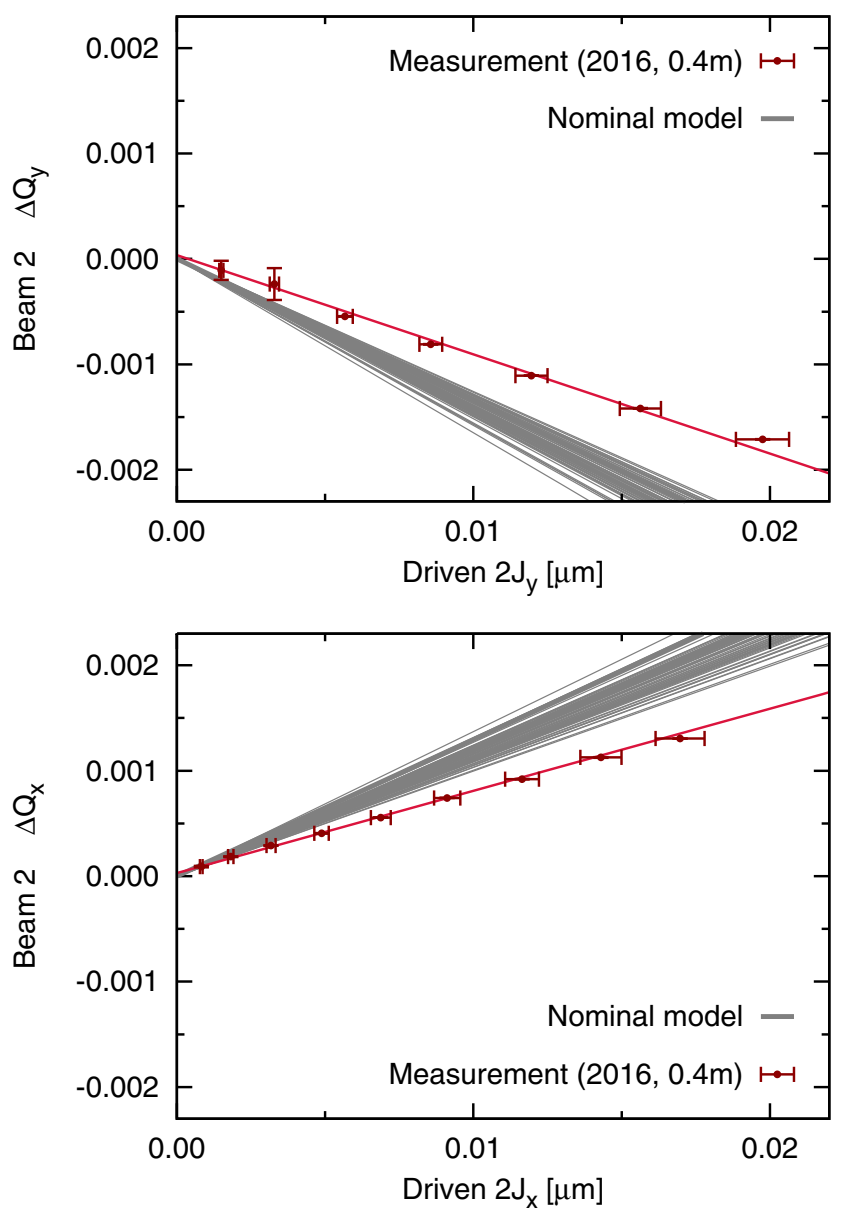

FIG. 5. Amplitude detuning measured with flat orbit in 2016, for LHC Beam 2 at $\beta^{*}=0.4 \mathrm{~m}$ with Landau octupoles powered off (red). Simulated detuning is shown in gray, for models based upon the measured magnetic errors. Sixty instances of the magnetic errors are simulated, corresponding to the uncertainty in the magnetic measurements.

Fig. 5. Direct terms $\left(\frac{\partial Q_{x}}{\partial \epsilon_{x}}\right.$ and $\left.\frac{\partial Q_{y}}{\partial \epsilon_{y}}\right)$ showed a $\sim 30 \%$ discrepancy relative to magnetic measurements. On their own these measurements cannot be used to determine corrections, as it is impossible to distinguish sources in IP1 and IP5. When comparing measurements of feed-down to tune as a function of crossing angle in IP1 however, it was found that the quadratic tune shift $\left(\Delta Q \propto y^{2}\right.$, dependent on IR-octupole errors) consistently showed a good agreement with predictions of the magnetic model [49]. An example is shown in Fig. 6. In contrast quadratic variation of tune as a function of crossing angle in IR5 (CMS) showed substantial discrepancies with the predictions of the magnetic model [49].

By combining detuning and feed-down data a correction strategy could be devised. Given the close agreement to model predictions, IR1 (ATLAS) corrections were calculated directly from the magnetic measurements (following [47]). Correctors left and right of IP5 were then matched in simulation to minimize the residual detuning expected for 


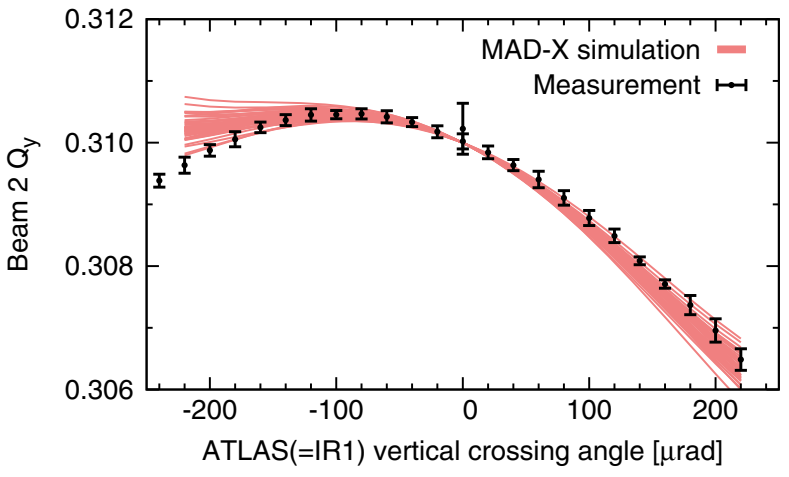

FIG. 6. Measured tune (black) as a function of the vertical crossing angle in the ATLAS (IR1) insertion, compared to predictions based upon magnetic measurements (red).

both beams after application of the IR1 (ATLAS) correction. Figure 7 shows the applied beam-based corrections left and right of IR1 (ATLAS) and IR5 (CMS), compared to the corrections expected from the magnetic measurements. The discrepancy with the magnetic model remains unexplained, though feed-down of decapole or dodecapole errors due to alignment errors in the IRs appears one potential source [59].

A beneficial effect from this correction was seen immediately, through a clear improvement in the performance of beam instrumentation. Figure 8 shows an example of substantial reduction in noise in the online tune measurement, obtained upon application of the normal octupole correction.

A clear influence was also observed in the online measurement of linear coupling via the LHC base-band tune (BBQ) system [60,61]. A substantial reduction $\left(\Delta\left|C^{-}\right| \approx 4 \times 10^{-3}\right)$ can be seen in Fig. 9 upon application of the normal octupole correction.

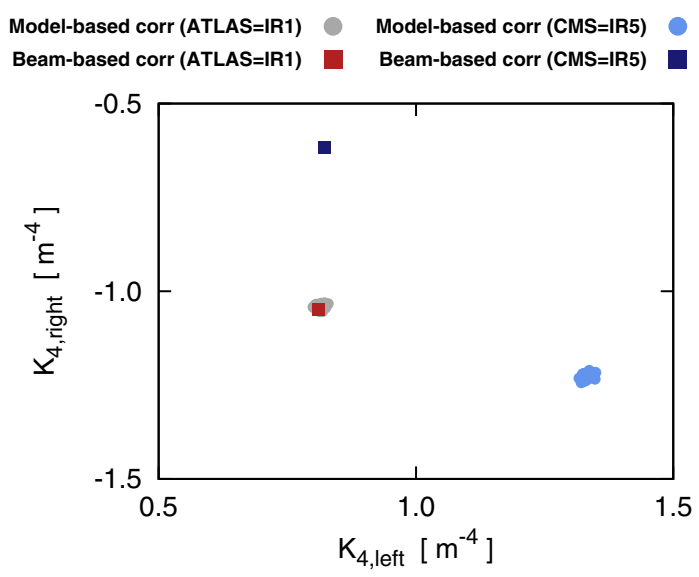

FIG. 7. Beam-based correction for normal octupole errors in the ATLAS (IR1) and CMS (IR5) insertions, compared to expected corrections based upon magnetic measurement. Sixty instances of model-based corrections are shown corresponding to the uncertainty in the magnetic measurements.

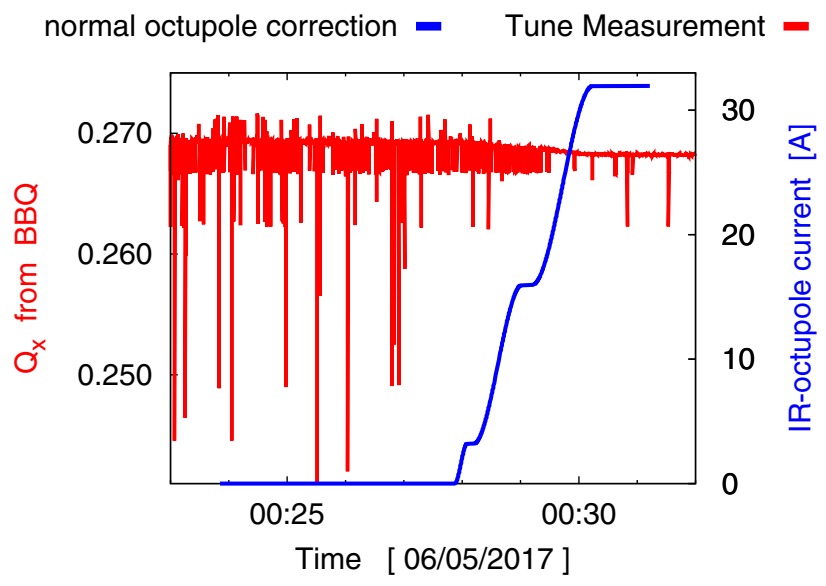

FIG. 8. Improvement in online tune measurement quality upon application of corrections for normal octupole errors in the ATLAS (IR1) and CMS (IR5) insertions.

It should be emphasized that the coupling shift in Fig. 9 is not real. In 2012 it was observed that the LHC BBQ displayed impossibly large coupling shifts upon changes in octupole powering [62], incompatible with more reliable measurements. Online measurement of $\left|C^{-}\right|$could only be trusted in the absence of strong octupolar sources [63] (including in the IRs). This artifact was demonstrated to arise from changes in the signal-to-noise ratio of coupling lines in the BBQ spectrum, due to increased tune-spread generated by octupoles [64]. The apparent shift in Fig. 9 corresponds to an improved reliability of the BBQ, due to a reduction in tunespread upon application of the correction. Indeed, after IRoctupole correction the $\left|C^{-}\right|$recorded by the BBQ was found to be consistent with that measured via the more reliable acdipole technique $\left(\left|\mathrm{C}^{-}\right|_{\text {ac-dipole }}=0.0028\right)$, facilitating continuous $\left|C^{-}\right|$measurement during the squeeze.

The improved BBQ performance was of significant assistance to LHC operation. Within the context of optics

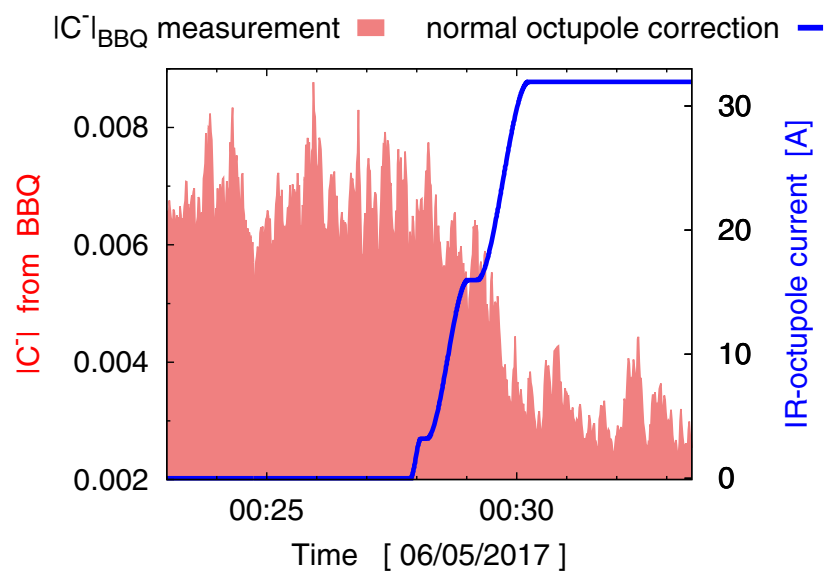

FIG. 9. Improvement in online coupling measurement upon application of normal octupole corrections in the ATLAS (IR1) and CMS (IR5) insertions. 


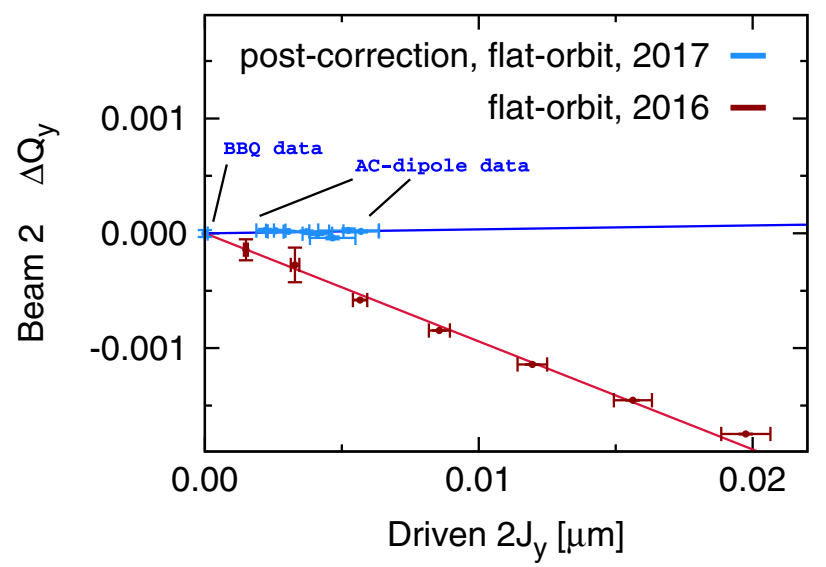

FIG. 10. Example of amplitude detuning measurements at $\beta^{*}=0.3 \mathrm{~m}$ after IR-octupole correction (blue), and $\beta^{*}=$ $0.4 \mathrm{~m}$ before correction (red).

commissioning this improved performance was also fundamental to obtain high quality K-modulation data at $\beta^{*}=0.4$ and $0.3 \mathrm{~m}$, facilitating control of the $\beta^{*}$ imbalance between the ATLAS and CMS experiments. The observation also provided a qualitative validation of the normal octupole correction, which remained in place throughout commissioning and operation in 2017 and 2018.

Direct confirmation of the correction was performed at the end of the 2017 commissioning period, at $\beta^{*}=0.3 \mathrm{~m}$. Figure 10 shows a the amplitude detuning measured with ac-dipole at $\beta^{*}=0.3 \mathrm{~m}$ after normal octupole correction in IR1 and IR5 (blue), compared to that measured at $\beta^{*}=$ $0.4 \mathrm{~m}$ before correction (red). Due to a reduced physical aperture at $\beta^{*}=0.3 \mathrm{~m}$ and larger emittance beams, the amplitude range probed after correction is significantly smaller than was possible before correction. By combining ac-dipole measurements with BBQ $[60,61]$ data for the unperturbed tune however, it was still possible to obtain a

TABLE II. Amplitude detuning coefficients at $\beta^{*}=0.4 \mathrm{~m}$ without normal octupole correction, and at $\beta^{*}=0.3 \mathrm{~m}$ after correction.

\begin{tabular}{lccc}
\hline \hline $\begin{array}{l}\text { Detuning coefficients } \\
{\left[10^{3} \mathrm{~m}^{-1}\right]}\end{array}$ & $\begin{array}{c}\beta^{*}=0.4 \mathrm{~m} \\
\text { (no correction) }\end{array}$ & $\begin{array}{c}\beta^{*}=0.3 \mathrm{~m} \\
\text { (with correction) }\end{array}$ \\
\hline LHCB1 & $\frac{\partial Q_{x}}{\partial \epsilon_{x}}$ & $43 \pm 1$ & $-3 \pm 1$ \\
& $\frac{\partial Q_{x}}{\partial \epsilon_{y}}=\frac{\partial Q_{y}}{\partial \epsilon_{x}}$ & $0 \pm 1$ & $5 \pm 3$ \\
& $\frac{\partial Q_{y}}{\partial \epsilon_{y}}$ & $-50 \pm 1$ & No measurement \\
LHCB2 & $\frac{\partial Q_{x}}{\partial \epsilon_{x}}$ & $38 \pm 1$ & $-2 \pm 1$ \\
& $\frac{\partial Q_{x}}{\partial \epsilon_{y}}=\frac{\partial Q_{y}}{\partial \epsilon_{x}}$ & $1 \pm 1$ & $-3 \pm 2$ \\
& $\frac{\partial Q_{y}}{\partial \epsilon_{y}}$ & $-44 \pm 1$ & $2 \pm 1$ \\
\hline \hline
\end{tabular}

good measurement of amplitude detuning. Table II details the detuning coefficients obtained for the two cases. Amplitude detuning generated in the experimental IRs scales with $\left(\beta^{*}\right)^{-2}$ [65]. Comparing the pre- and postcorrection measurements, the small postcorrection detuning at $\beta^{*}=0.3 \mathrm{~m}$ is thus obtained in spite of the expected increase in amplitude detuning as the beams are squeezed from 0.4 to $0.3 \mathrm{~m}$.

Application of normal octupole corrections in the ATLAS (IR1) and CMS (IR5) insertions substantially reduced the direct detuning terms, with minimal disruption to the already small cross-term detuning. One detuning term for Beam $1\left(\frac{\partial Q_{y}}{\partial \epsilon_{y}}\right)$ could not be measured after correction, however as both errors and correctors are common to the two beams it may be confidently constrained to the same level as the measured detuning coefficients after correction. The observed reduction in amplitude detuning provides a direct validation of the global quality of the correction, and achieves one of the main aims of the nonlinear optics commissioning: removing the IR-contribution to the tune-spread in the squeeze. The small level of residual detuning and the stability of the correction/errors was also revalidated over larger amplitude ranges in late 2017 [66] and early 2018 [59].

The local nature of the normal octupole correction can be confirmed by examining feed-down to tune as a function of crossing angle. Figure 11 compares the uncorrected $Q_{y}$ dependence on the vertical crossing angle in IP1, to that observed after normal octupole correction in 2017. Application of the normal octupole correction resulted in a substantially more linear tune shift as a function of the applied closed orbit distortion in the IR. Reductions to the quadratic variation of tune with crossing angle were observed in both IPs.

Direct RDT measurement was not used in calculation of the correction. Using the techniques developed in $[55,56]$ however, some ac-dipole RDTs could be observed, which provided further validation of the normal octupole correction. In particular, correction significantly

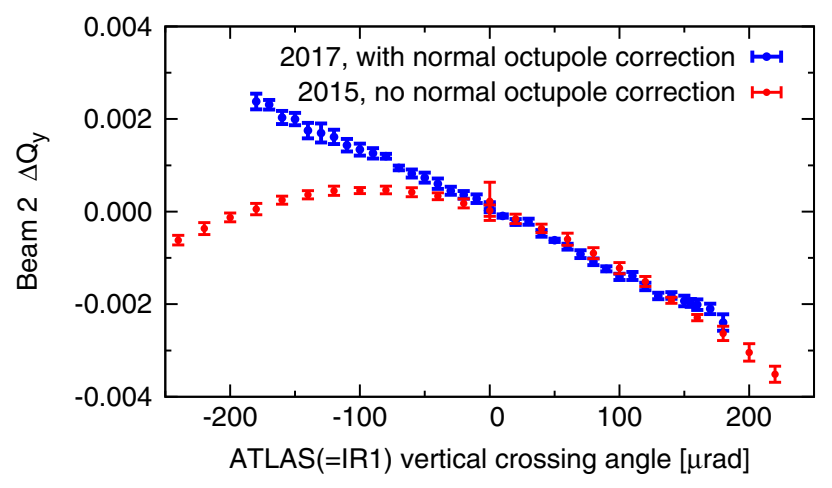

FIG. 11. Example of tune shift with crossing angle with (blue) and without (red) correction of normal octupole errors. 


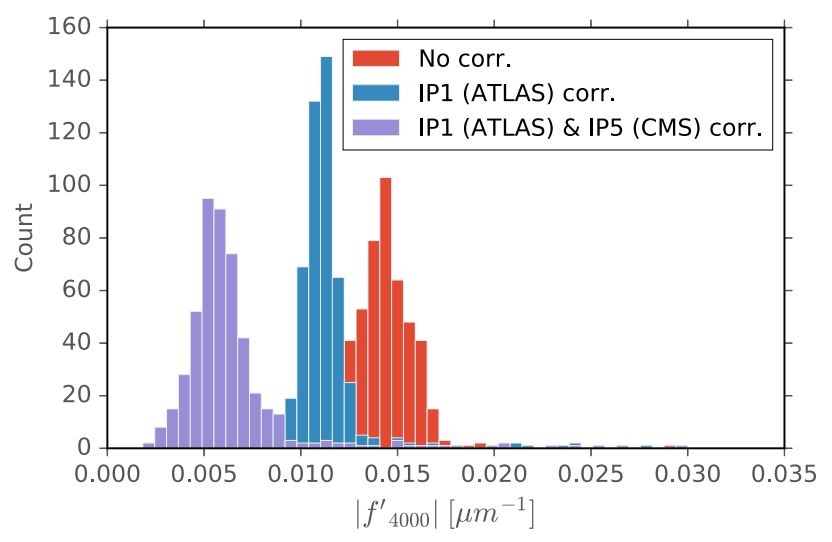

FIG. 12. Histogram of the $\left|f_{4000}^{\prime}\right|$ ac-dipole resonance driving term, related to the $4 Q_{x}$ resonance, measured in LHC BPMs with and without correction of normal octupole errors in the ATLAS (IR1) and CMS (IR5) insertions.

reduced the strength of the $4 Q_{x}$ resonance. This is seen in Fig. 12, which shows histograms of the $\left|f_{4000}^{\prime}\right|$ RDT measured in the LHC BPMs with and without correction of the normal octupole errors in the ATLAS and CMS
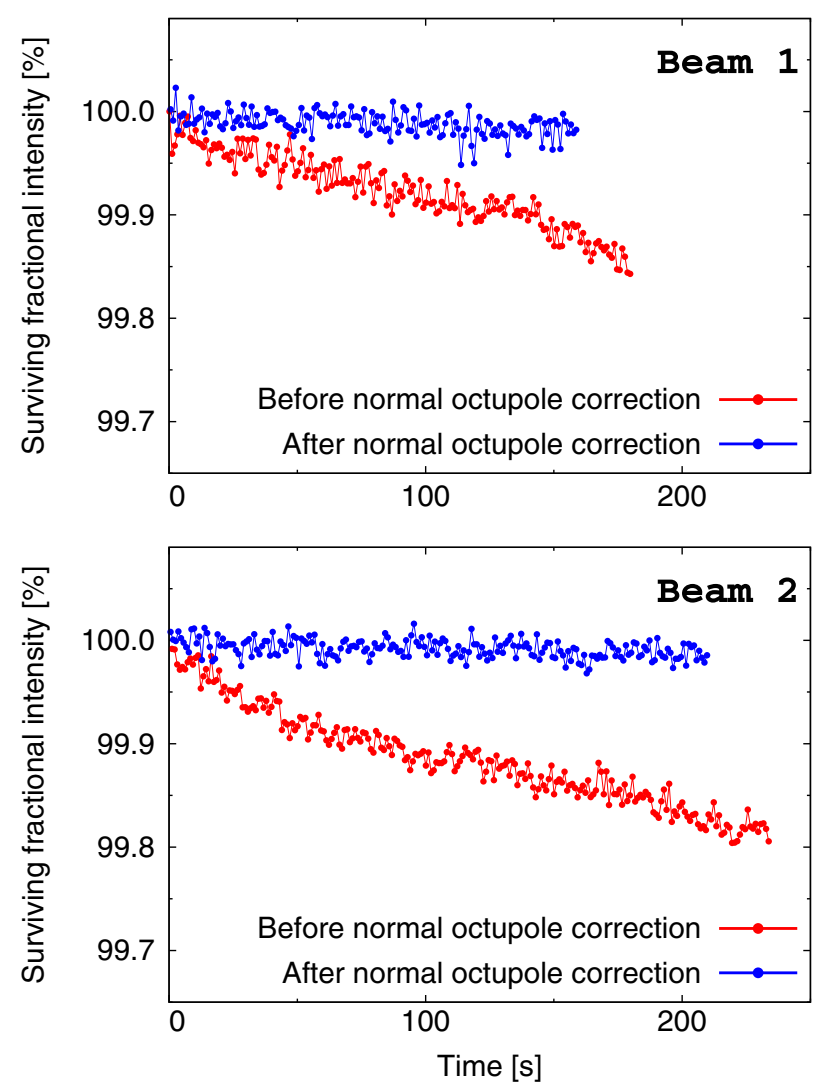

FIG. 13. Change in fractional intensity determined from BeamCurrent Transformer data, for the two minutes prior (red), and following (blue), application of normal octupole corrections in IR1 (ATLAS) and IR5 (CMS). Measurements performed at $\beta^{*}=$ $0.14 \mathrm{~m}$ during dedicated tests of the ATS optics $[67,68]$. insertions ( $f^{\prime}$ indicates a RDT for driven motion with an ac-dipole [54]). The quantity shown is the amplitude of the driven RDT.

Application of the normal octupole correction was also found to substantially increase beam lifetime in machine studies to test the $\beta^{*}$ reach of the LHC. At $\beta^{*}=0.14 \mathrm{~m}$, it was found that lifetime was so low as to inhibit linear optics measurement and correction. In response the IR correction was applied. Figure 13 shows the change in fractional intensity recorded for the two minutes before (red) and after (blue) application of the normal octupole correction.

\section{B. Normal sextupole correction in the CMS (IR5) insertion}

Due to the horizontal orientation of the crossing angle plane in the CMS experimental insertion (IR5), normal sextupole errors feed-down to generate a normal quadrupole perturbation. The errors may be examined by considering the linear variation of tune with the horizontal crossing angle in IR5 (CMS). Figure 14 (red) shows the measured tune-shift as a function of the applied crossing angle bump for LHC Beam 1. A large asymmetry is observed between the linear variation of $Q_{x}$ and $Q_{y}$ with crossing angle, with sextupolar feed-down mainly perturbing the horizontal plane of Beam 1. Similar results were obtained for Beam 2, with feed-down mainly influencing the vertical plane. Some residual quadratic variation of tune with crossing angle can also be observed to remain after normal octupole correction.

In order to compensate for normal sextupole errors, linear variation of tune with crossing angle (determined from second-order polynomial fits) was matched in MAD-X, using the normal sextupole corrector circuits left and right of IR5 (CMS). Settings of these common correctors which reproduced the linear tune shift of both beams were reversed and applied in the accelerator. Blue data in Fig. 14 shows the result of a crossing angle scan performed after correction. The gray line shows the expected tune change after correction. The linear component of the tune shift was substantially reduced, and the observed variation after correction agrees well with expectation. Figure 15 compares the applied beam-based correction to the expectation from magnetic measurements. A discrepancy exists between beam- and magnetic-measurements. This is not unexpected as discrepancies between simulated and measured feeddown were already observed [49]. The discrepancy remains to be understood, however it was also observed in earlier beam-based studies that misalignments of the normal octupole correctors could generate additional feed-down capable of influencing the lower order corrections [49]. Such effects may offer a potential explanation, but would require additional measurements to confirm.

During luminosity production the LHC operates very close to the linear coupling $\left(Q_{x, \text { frac }}-Q_{y \text {,frac }}=0\right)$ resonance, with fractional tune separation in the range 


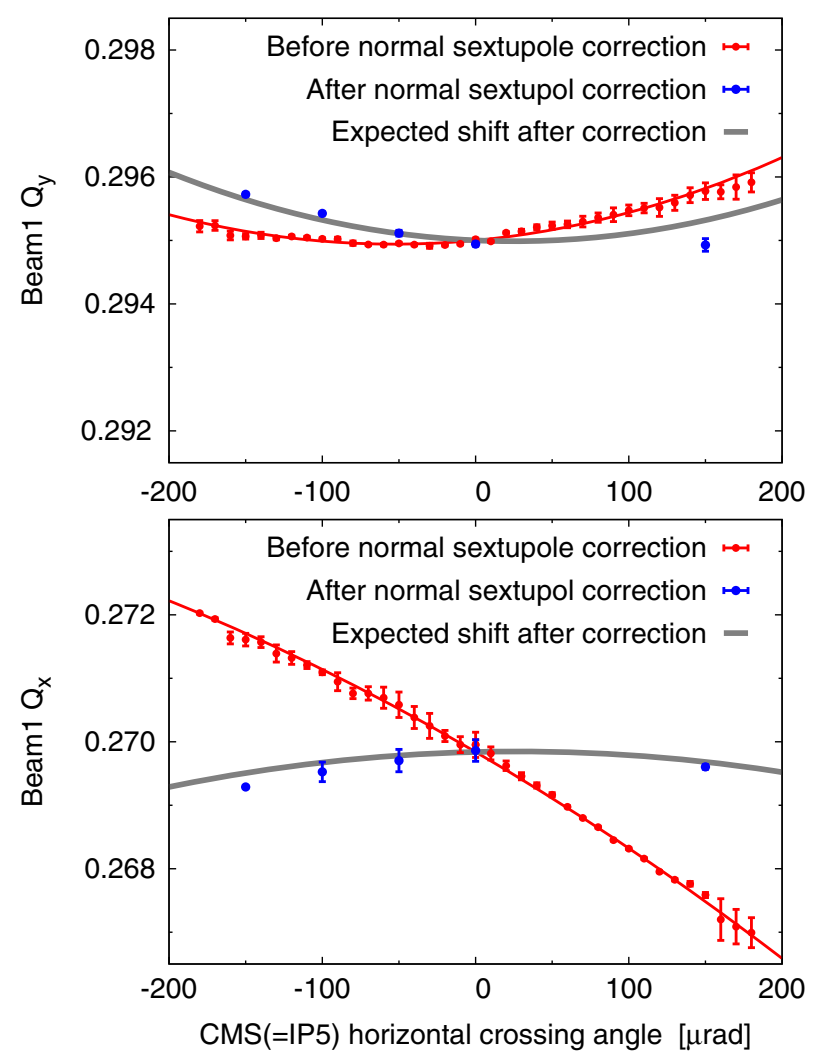

FIG. 14. Tune shift of LHC Beam 1 as a function of the horizontal crossing angle in CMS (IR5). Red data show the tune shift measured after application of normal octupole corrections, but before correction of normal sextupole. The fit to the measured data is also shown in red. Blue data corresponds to the tune shift measured after application of normal sextupole corrections. The gray line indicates the expected variation of tune after normal sextupole correction.

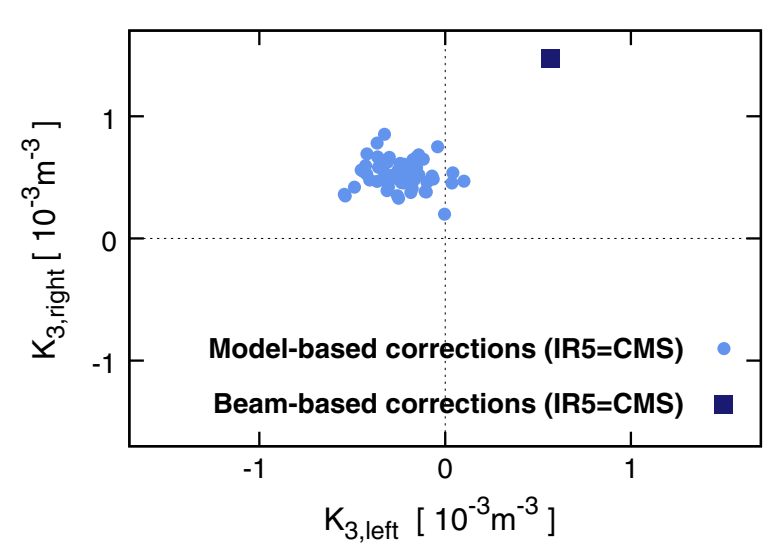

FIG. 15. Beam-based corrections for the normal sextupole errors applied in the CMS (IR5) insertion, compared to the expected corrections based upon magnetic measurements. Sixty instances of the model-based corrections are shown, corresponding to the uncertainty in the magnetic measurements.
$\Delta Q_{\text {frac }}=0.01-0.004$. Table III details the changes to fractional tune separation expected during crossing angle leveling due to feed-down in IR5 (CMS). Prior to normal sextupole correction, reduction of the IR5 (CMS) crossing angle from 150 to $100 \mu \mathrm{rad}$ during luminosity leveling would give $\mathrm{a} \sim 30 \%$ reduction to the tune separation at $\beta^{*}=$ $0.4 \mathrm{~m}$ (reaching $\sim 40 \%$ at $\beta^{*}=0.3 \mathrm{~m}$ ). Application of the normal sextupole correction therefore represents a very significant improvement to tune stability during luminosity production. This is critical to maintaining Landau damping (which is dependent on linear coupling in relation to tune separation [32,33]) as well as being potentially significant for beam lifetime (tune shifts due to feed-down from IRnonlinear errors can generate transient beam losses [68] as well as shift the working point to suboptimal regions of the tune diagram).

While reduction of tune shift with crossing angle is of operational benefit, the main concern in relation to normal sextupole errors in the IRs is perturbation of linear optics. Figure 16 shows histograms of the change in beta-beating at $\beta^{*}=0.4 \mathrm{~m}$ between $\pm 150 \mu \mathrm{rad}$ crossing angles, as measured in the BPMs around the LHC ring. This differential beta-beat due to sextupolar feed-down (before correction, red) is non-negligible in relation to the LHC's stated aim of achieving a $1 \%$ tolerance on the beta-beating [7], and will increase for smaller $\beta^{*}$. This contribution had never previously been considered. The optics quality obtained during previous years' commissioning with flat closed orbit [6,7] thus represents a slight underestimate of the true $\beta$-beat during operation for luminosity production, where crossing angles are applied. After minimization of linear tune variation with crossing angle using the correctors in IR5, Fig. 16 (blue) shows a pronounced improvement to the distribution of the differential beta-beat vs crossing angle measured in the LHC BPMs. Betabeating in the vertical plane of Beam 1 and horizontal plane of Beam 2 was relatively stable before correction (comparable to Fig. 16 after correction), and was unaffected by the sextupole trims.

TABLE III. Change to tune separation during LHC crossing angle leveling, due to feed-down in the CMS (IR5) insertion before and after correction of normal sextupole errors. Values quoted assume that linear coupling is small in relation to the tune separation. Values in [\%] are quoted relative to the minimum tune separation used during luminosity production in 2017.

\begin{tabular}{|c|c|c|}
\hline & $\begin{array}{c}\text { Before corr } \\
{\left[10^{-3}\right]}\end{array}$ & $\begin{array}{c}\text { After corr } \\
{\left[10^{-3}\right]}\end{array}$ \\
\hline LHCB1 $\Delta\left|Q_{x, \text { frac }}-Q_{y, \text { frac }}\right|$ & $-1.22 \pm 0.01$ & $-0.32 \pm 0.02$ \\
\hline $\operatorname{LHCB} 1 \frac{\Delta\left|Q_{x, \text { frac }}-Q_{y, \text { frac }}\right|}{\left|Q_{x, \text { frac }}-Q_{y, \text { frac }}\right|}$ & $-30 \%$ & $-7 \%$ \\
\hline LHCB2 $\Delta\left|Q_{x, \text { frac }}-Q_{y, \text { frac }}\right|$ & $-0.80 \pm 0.01$ & $-0.44 \pm 0.01$ \\
\hline LHCB2 $\frac{\Delta\left|Q_{x, \text { frac }}-Q_{y, \text { frac }}\right|}{\left|Q_{x, \text { frac }}-Q_{y, \text { frac }}\right|}$ & $-20 \%$ & $-10 \%$ \\
\hline
\end{tabular}



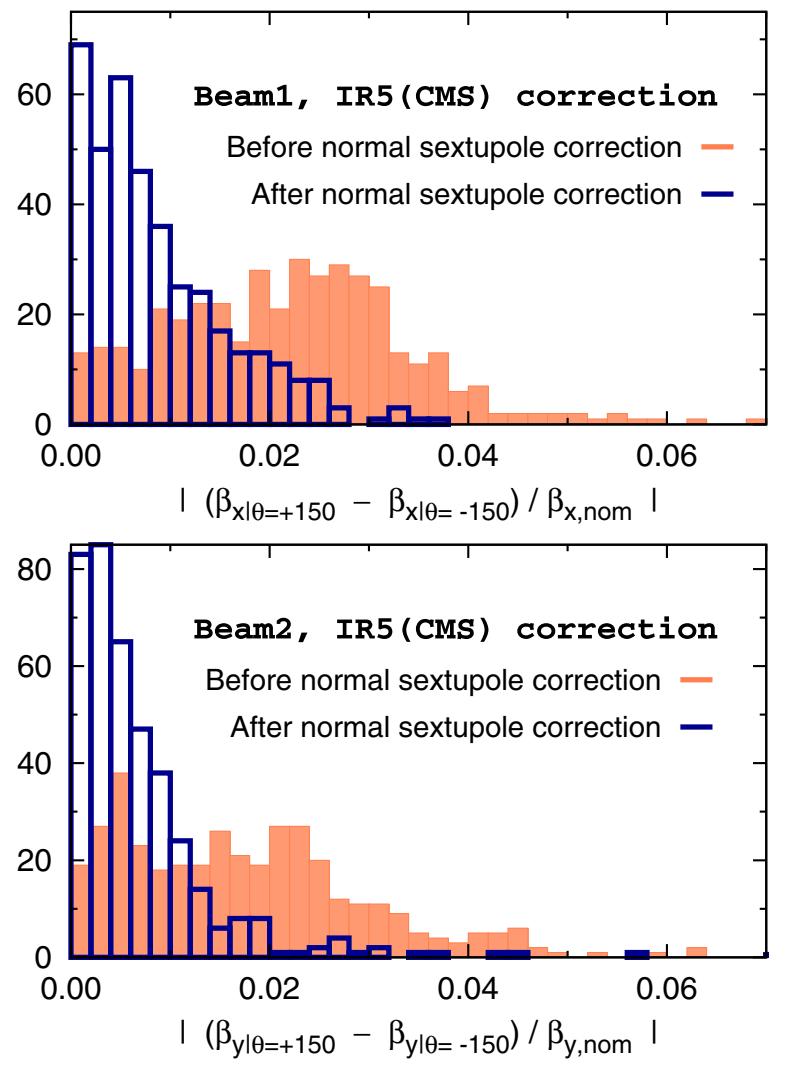

FIG. 16. Histograms of Beam 1 (top) and Beam 2 (bottom) beta-beating measured at LHC BPMs, before (blue) and after (red) normal sextupole compensation in IR5 (CMS).

\section{Normal sextupole, skew sextupole, and skew octupole correction in the ATLAS (IR1) insertion}

In contrast to the CMS (IR5) insertion, the crossing plane for the ATLAS (IR1) experiment is orientated vertically. In IR1 therefore, normal sextupole errors feeddown to generate a linear coupling source which varies linearly as function of the applied crossing angle. Note that "linear coupling" in this context refers to an action independent coupling between the transverse planes via a skew-quadrupolelike perturbation, which will drive the $\left(Q_{x}+Q_{y}\right)$ and $\left(Q_{x}-Q_{y}\right)$ resonances and generate a closest approach of the fractional tunes $\left(\left|C^{-}\right|=\Delta Q_{\min }\right)$ [69]. Skew octupole errors feed-down both horizontally and vertically to generate linear coupling which varies quadratically as a function of the orbit bump. In IR5 (CMS) coupling shifts going from 0 to $150 \mu \mathrm{rad}$ (the operational crossing angle for IR1 and IR5 in 2017) were comparatively small. In the ATLAS (IR1) insertion large coupling shifts were seen, motivating correction of normal sextupoles and skew octupoles in this IR.

To correct the feed-down ac-dipole measurements were performed as a function of crossing angle, obtaining $f_{1001}$ RDTs at all BPMs. A setting of normal sextupole and skew
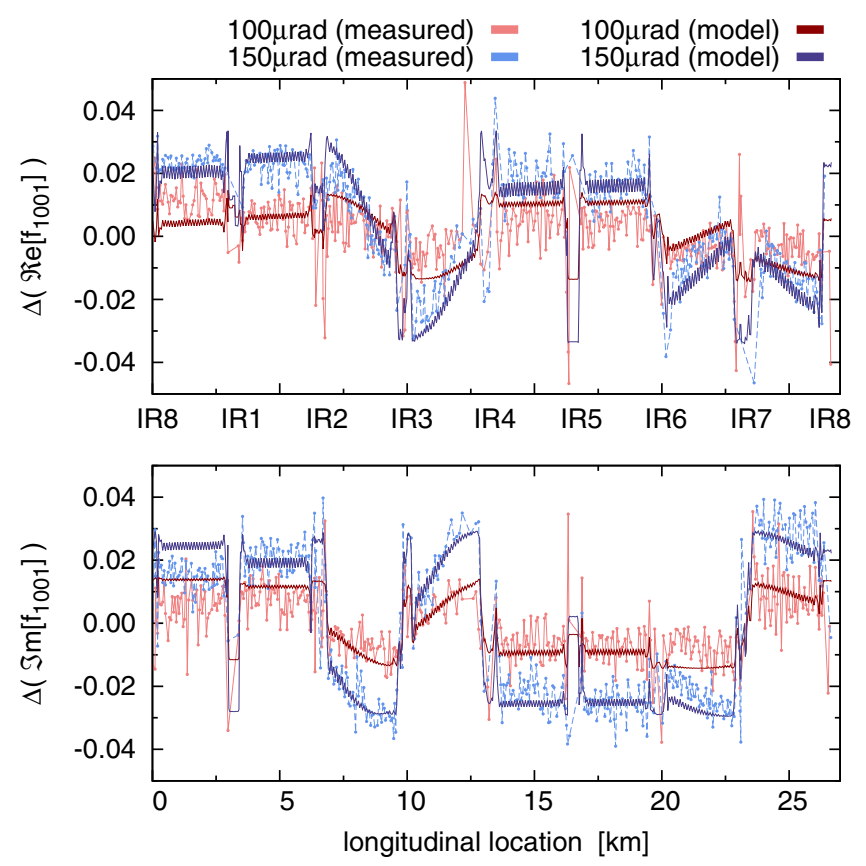

FIG. 17. Measurement of $f_{1001}$ at 100 (light red) and $150 \mu \mathrm{rad}$ (light blue) crossing angles. The values plotted are the difference of the real (top) and imaginary (bottom) parts of the RDT with respect to measurement at flat orbit, for all BPMs around the ring. Simulated RDT shifts for a model which includes normal sextupole and skew octupole corrector powering which best reproduced the crossing angle scan are shown (dark red/dark blue).

octupole correctors was found in simulation which reproduced the shift vs crossing angle of the real and imaginary parts of $f_{1001}$ relative to flat orbit. An example of this is shown in Fig. 17 for two of the measured crossing angles. This setting was inverted and applied in the LHC to minimize the feed-down.

To characterize the feed-down before and after correction the required global coupling correction needed to minimize $\left|C^{-}\right|$is considered. In the LHC global coupling is compensated via a pair of skew-quadrupole knobs which are defined to act orthogonally on the $\Re e$ and $\Im m$ parts of the RDT at a specific point on the ring (this is described in detail in [70]). Knob strengths are expressed in units of the $\left|C^{-}\right|=\Delta Q_{\min }$ shift each would generate independently, such that

$$
\left|C^{-}\right|=\sqrt{\mathrm{CORR}_{\Re e}^{2}+\mathrm{CORR}_{\Im m}^{2}}
$$

where $\mathrm{CORR}_{\Re i e}$ and $\mathrm{CORR}_{\Im m}$ are the knob strengths. It is in general advantageous to separate the coupling into real and imaginary shifts in this way since the linear and quadratic evolution with crossing angle can be easily discerned. Figure 18 shows the required global coupling correction of Beam 2 as a function of crossing angle before 
and after correction. Large linear and quadratic shifts due to sextupole and skew-octupole feed-down were observed. Correction was performed via two iterations in 2017 and 2018, further details are provided in [34]. Measurements shown before correction (red) are from 2017 at $\beta^{*}=0.4 \mathrm{~m}$. Measurements after correction (green) were performed in 2018 at $\beta^{*}=0.25 \mathrm{~m}$. Coupling from skew-quadrupole fields in the IR scales with $\sim \sqrt{\beta_{x}^{*} \beta_{y}^{*}}$. The gray fit in Fig. 18 shows the extrapolated post-correction feed-down at $\beta^{*}=0.4 \mathrm{~m}$. Comparable improvements were obtained for Beam 1 over the two commissioning periods [34].

A substantial improvement to coupling stability with crossing angle was obtained through correction of the normal sextupole and skew octupole errors in the ATLAS (IR1) insertion. This is of particular relevance to LHC operation with crossing angle luminosity leveling, since a $\left|C^{-}\right|$comparable to the fractional tune separation risks loss of Landau damping [33]. Table IV details the coupling shifts which could be generated during crossing angle leveling from $150 \mu \mathrm{rad} \rightarrow 100 \mu \mathrm{rad}$ before and after correction of normal-sextupole and skew-octupole errors in IR1 (ATLAS). Pre-correction shifts were large enough to pose an operational challenge to Landau damping, and

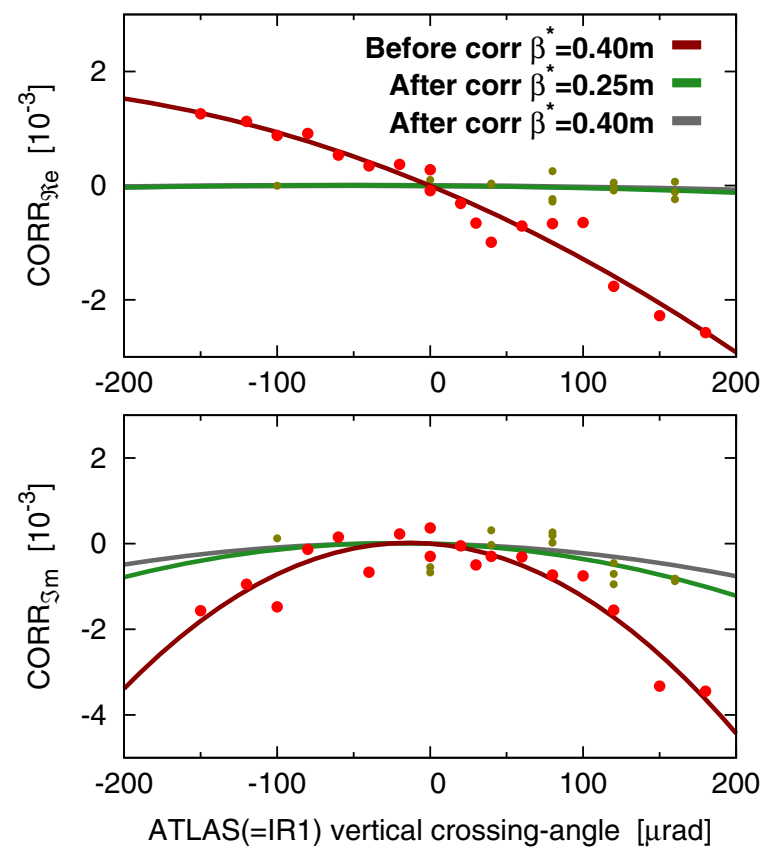

FIG. 18. Required global coupling correction [70] of Beam 2 as a function of vertical crossing angle in the ATLAS (IR1) insertion. Measurements before normal sextupole and skew octupole correction are for $\beta^{*}=0.4 \mathrm{~m}$ (red), and after correction for $\beta^{*}=0.25 \mathrm{~m}$ (green). The postcorrection measurement extrapolated to $\beta^{*}=0.4 \mathrm{~m}$ is shown (gray). The correction strength is defined as $\left|C^{-}\right|=\sqrt{\mathrm{CORR}_{\Re e}^{2}+\mathrm{CORR}_{\Im m}^{2}}$ where $\mathrm{CORR}_{\Re e}$ and $\mathrm{CORR}_{\Im m}$ are skew-quadrupole knobs acting orthogonally on the real and imaginary parts of $f_{1001}$ at a specific location in the ring [70].
TABLE IV. Maximum possible change to $\left|C^{-}\right|$which can be generated via normal-sextupole/skew-octupole feed-down in IR1 (ALTAS) during crossing angle leveling. Values for $\beta^{*}=0.3 \mathrm{~m}$ are based on the expected scaling of the feed-down with $\beta^{*}$. The largest possible value from either Beam 1 or Beam 2 is shown.

\begin{tabular}{lccc}
\hline \hline & \multicolumn{2}{c}{$\Delta\left|C^{-}\right|\left[10^{-3}\right]$} & $\frac{\Delta\left|C^{-}\right|}{Q_{x, \text { frac }}-Q_{y, \text { frac }}}$ \\
\cline { 2 - 4 } & $\beta^{*}=0.4 \mathrm{~m}$ & $\beta^{*}=0.3 \mathrm{~m}$ & $\beta^{*}=0.3 \mathrm{~m}$ \\
\hline No correction & $\leq 1.5$ & $\leq 2.0$ & $\leq 50 \%$ \\
After correction & $\leq 0.4$ & $\leq 0.6$ & $\leq 15 \%$ \\
\hline
\end{tabular}

nonlinear correction has significantly reduced the associated operational risks.

Figure 19 compares the beam- and magnetic-model-based correction for normal sextupole errors in the ALTAS(IR1) insertion. As with the CMS (IR5) correction discrepancies with the magnetic model are apparent. Once again no definitive explanation for the discrepancy can be offered, though alignment errors of the higher-order correctors remain a possible candidate. Table $\mathrm{V}$ details the applied skew octupole correction, together with the expectation from magnetic measurements. The skew octupole corrector left of the IR is broken in the LHC, and minimization of the feeddown to coupling was performed using the single available corrector. The beam- and model- based corrections cannot be compared directly therefore, however the magnitude and sign of the beam-based correction is comparable to that expected from the magnetic model.

Correction of skew-octupolar feed-down to coupling is viable using a single corrector. Direct correction of the skew-octupole resonances however, requires two functioning correctors [47]. Consequently the applied correction cannot represent an optimal compensation of the skewoctupole resonances. It was found that the coupling-based correction reduced the strength of the $f_{1210}^{\prime}$ RDT driving the $Q_{x}-Q_{y}$ resonance in Beam 2, while increasing the

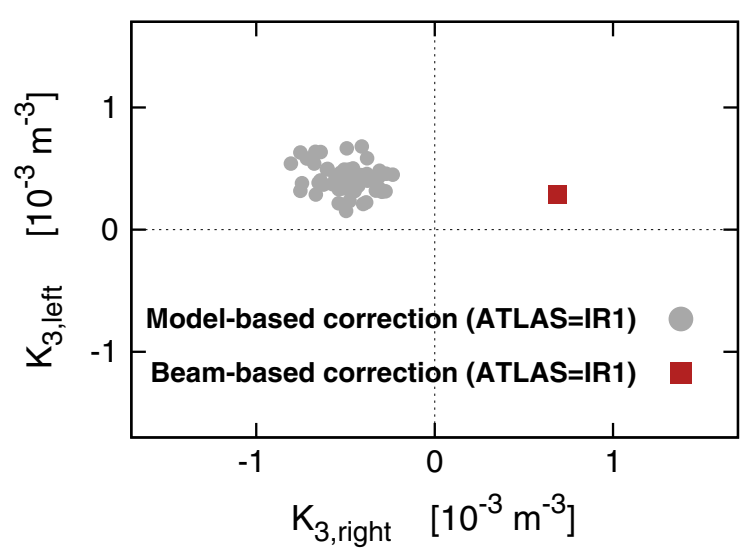

FIG. 19. Beam- and model-based correction for normal sextupole errors in the ATLAS IR (IR1), compared to the expected corrections based upon magnetic measurements. 
TABLE V. Beam- and model- based correction strength for skew octupole errors in IR1 (ATLAS). Note that the skew octupole corrector right of IR1 is broken and is not considered in the beam-based correction.

\begin{tabular}{lcc}
\hline \hline & Beam-based & Model-based \\
\hline Left of IR1 (ATLAS) $\left[\mathrm{m}^{-4}\right]$ & Broken & $+0.81 \pm 0.04$ \\
Right of IR1 (ATLAS) $\left[\mathrm{m}^{-4}\right]$ & +1.0 & $+0.12 \pm 0.05$ \\
\hline \hline
\end{tabular}

(initially smaller) RDT of Beam 1. Details of the RDT values before and after correction are given in Table VI. Given the emerging importance of skew-octupole sources directly to footprint distortion (and hence instabilities) in the LHC [30,71] alternative strategies based upon RDT compensation using the three available correctors in IR1 and IR5 are also being pursued [55,56].

In parallel with compensation of feed-down to linear coupling, skew sextupoles in IR1 (ATLAS) (which feeddown via the vertical IR1 crossing angle to generate normal quadrupole perturbations) were also compensated. Echoing normal sextupole correction in IR5 (CMS), skew sextupole correction in IR1 (ATLAS) was performed via minimization of the linear part of the tune shift as a function of the crossing angle. Figure 20 shows the feed-down to tune of LHC Beam 1 (left) and Beam 2 (right) before correction (red) and after correction (blue). The expected tune variation after correction is also shown in gray.

A significant improvement to tune shift vs crossing angle was obtained via skew-sextupole correction, however a clear discrepancy arose between the expected (gray) and obtained (blue) tune variation postcorrection. The discrepancy appeared for both planes of both LHC beams, and was not the result of tune drift. Rather, it resulted from parallel application of the skew-octupole correction for feed-down to coupling. Precorrection measurements (red) were performed with only normal octupole corrections (Sec. III A) applied. Postcorrection measurements (blue) were performed with corrections for skew sextupole, normal sextupole, and skew octupole applied. Skew octupoles feed-down to linear coupling, with a quadratic dependence on both the horizontal and vertical offset of the beam through the source $\left(\alpha \Delta x^{2}+\Delta y^{2}\right)$. In the case of a diagonal offset a skew octupole also generates feed-down to tune $(\propto \Delta x \Delta y)$. For a purely horizontal or vertical offset

TABLE VI. Strength of the $f_{1210}^{\prime}$ skew-octupole RDT before and after application of correction for skew octupole feed-down. Values are the average absolute resonance strength measured around the ring.

\begin{tabular}{lcc}
\hline \hline & \multicolumn{2}{c}{$\left\langle\left|f_{1210}^{\prime}\right|\right\rangle\left[\mu \mathrm{m}^{-1}\right]$} \\
\cline { 2 - 3 } & Beam 1 & Beam 2 \\
\hline Before correction & $0.37 \pm 0.02$ & $0.51 \pm 0.02$ \\
After correction & $0.45 \pm 0.02$ & $0.35 \pm 0.01$ \\
\hline \hline
\end{tabular}

therefore the skew-octupole correction should not influence the LHC tune, however a horizontal misalignment of the skew-octupole corrector would generate an additional source of linear tune variation as a function of the vertical crossing angle in IR1 (ATLAS). Figure 21 shows tune vs time as the skew octupole correction is turned on, for a vertical crossing angle of $150 \mu \mathrm{rad}$. A clear shift to tune was observed to $Q_{x, y}$ of both beams. Application of the skew-octupole correction in Fig. 21 was performed with a large tune separation $\left(\Delta Q_{x, y}=0.028\right)$, thus even though a significant coupling shift was generated the impact of $\Delta Q_{\min }$ on the tune is far smaller than observed in Fig. 21 (using [69]). Assuming therefore, that the tuneshifts measured in Fig. 21 are dominated by feed-down due to a constant horizontal offset of the beams through the corrector and the applied $150 \mu \mathrm{rad}$ vertical crossing angle, the expected linear variation of tune as a function of vertical crossing angle after skew-sextupole correction is modified, as shown by the green lines in Fig. 20, which are comparable to the observed change of tune.

The transverse offset of the skew-octupole corrector required to explain the observed tune shifts in Fig. 21, and the discrepancy between the expected (gray) and observed (blue) linear variation of tune in Fig. 20, were checked in simulation. A $1 \mathrm{~mm}$ horizontal misalignment toward the outside of the ring could explain the observed discrepancy for both beams (equivalently this could correspond to a closed-orbit error towards the inside of the ring). An alternative explanation is that cross-talk of the skewoctupole corrector with the skew-sextupole (which is nested in the same assembly) can modify its transfer function. In either case the additional linear tune variation can be corrected via an iteration of the skew sextupole on the right side of IP1. Such an iteration was not performed during commissioning of the $\beta^{*}=0.4 \mathrm{~m}$ optics in 2017 , but has since been applied to optics used for operation at $\beta^{*}<0.4 \mathrm{~m}$ in 2017 and 2018. Figure 22 shows the beam-based skew sextupole corrections before and after this iteration. The observation that feed-down and/or cross-talk from a higherorder correction spoiled sextupole compensation is significant for future LHC commissioning, and demonstrates the need for iterative compensation between multipole orders.

A slight improvement to linear optics stability versus crossing angle was observed upon application of the skew sextupole correction in IR1 (ATLAS), though less pronounced than that obtained via normal sextupole correction in IR5 (CMS). Potentially a consequence of additional beta-beat generated by feed-down from the skew octupole correction. Figure 23 shows the distribution of the relative change in $\beta$ measured around the LHC ring in the horizontal plane of Beam 1 and Vertical plane of Beam 2. Slight improvements can be discerned, particularly for Beam 2. The vertical plane of Beam 1 and horizontal plane of Beam 2 showed no noticeable change to the differential beta-beat. 

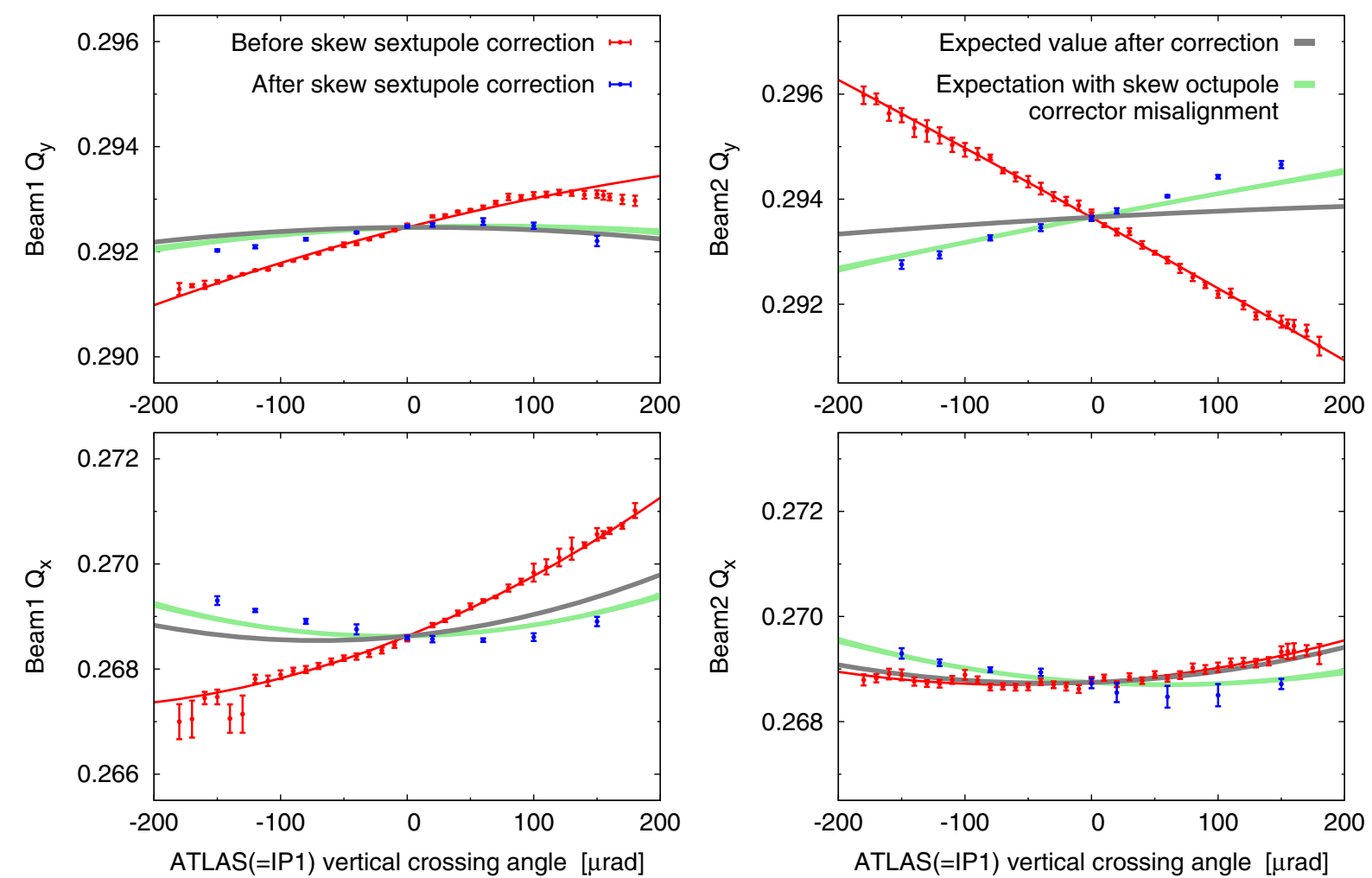

FIG. 20. Tune shift of LHC Beam 1 (left) and LHC Beam 2 (right) as a function of vertical crossing angle in IR1 (ATLAS). Red data shows the tune shift measured after application of normal octupole corrections, but before correction of normal/skew sextupole or skew octupole. The fit to the measured data is also shown in red. Blue data corresponds to the tune shift measured after application of normal/ skew sextupole, and skew octupole corrections. The gray line indicates the expected variation of tune after correction, based upon the red fit and expected performance of the skew sextupole correction. The green line represents the expected variation after correction, where the skew octupole corrector misalignment inferred from $Q$-shift during its application has been incorporated as a source of linear tune variation.

While only a limited improvement to linear optics was observed upon skew-sextupole compensation, the applied corrections were clearly beneficial to compensation of the $3 Q_{y}$ resonance (which is the most dangerous

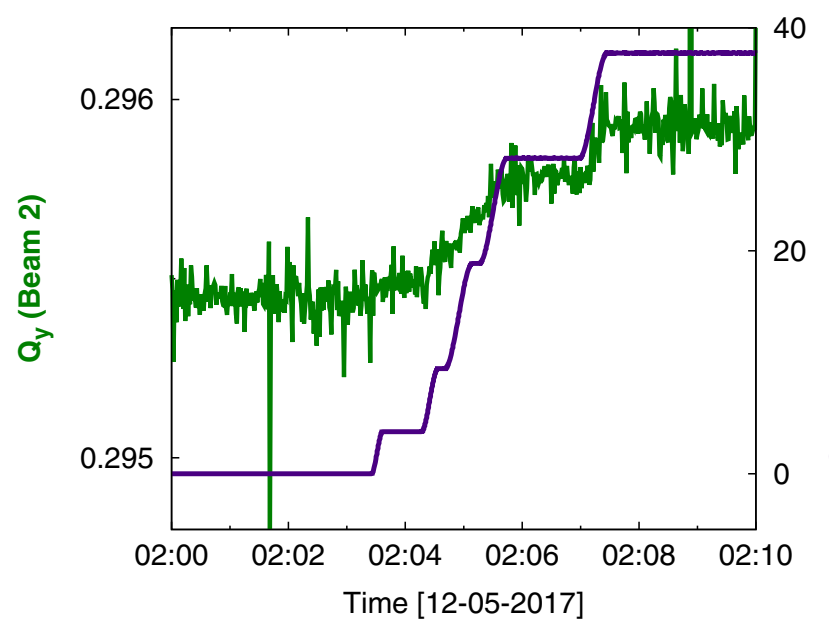

FIG. 21. Change to $Q_{y}$ of Beam 2 upon application of skewoctupole correction for feed-down to $\left|C^{-}\right|$, with a $150 \mu \mathrm{rad}$ vertical crossing angle in IR1 (ATLAS). skew-sextupole resonance relevant to LHC operation). Figure 24 shows histograms of the $\left|f_{0030}^{\prime}\right|$ measured around the ring before and after skew sextupole compensation, using the methods developed in $[55,56]$ (where $f^{\prime}$ indicates

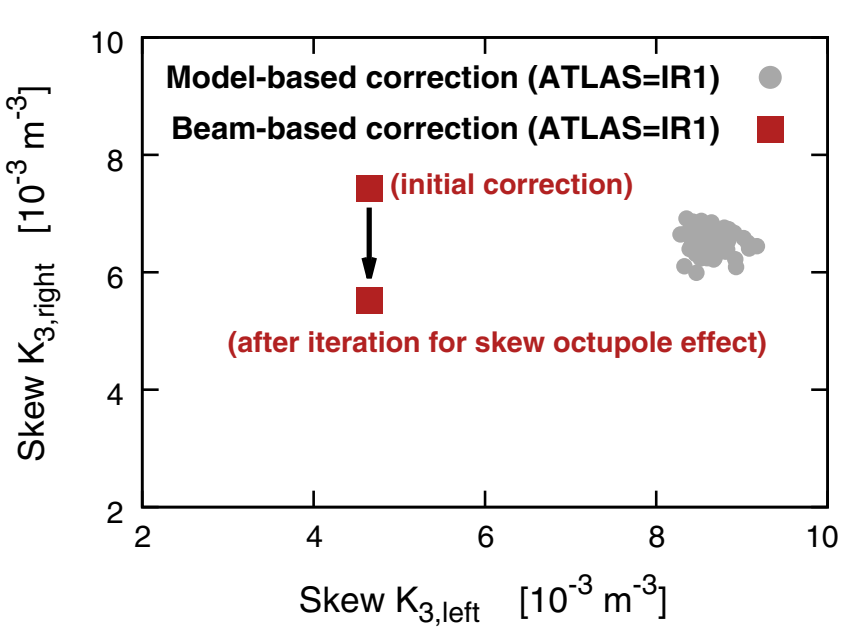

FIG. 22. Beam- and model-based correction of skew sextupole errors in the ATLAS (IR1) insertion. 

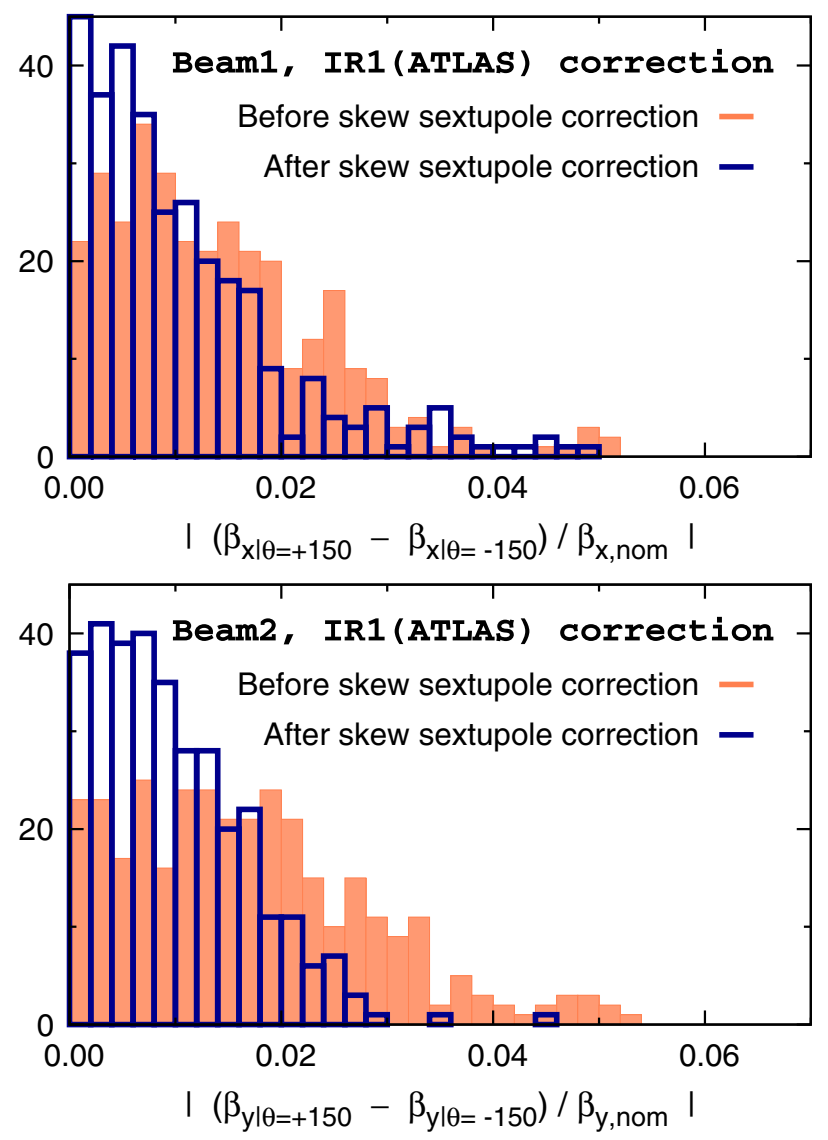

FIG. 23. Histograms of beta-beating measured at LHC BPMs, before (blue) and after (red) skew sextupole compensation in IR1 (ATLAS).

an RDT for driven motion with an ac-dipole [54]). Once again RDTs were used not to calculate corrections but to provide a validation of the correction calculated via feeddown. As with correction of normal sextupole errors in IR5

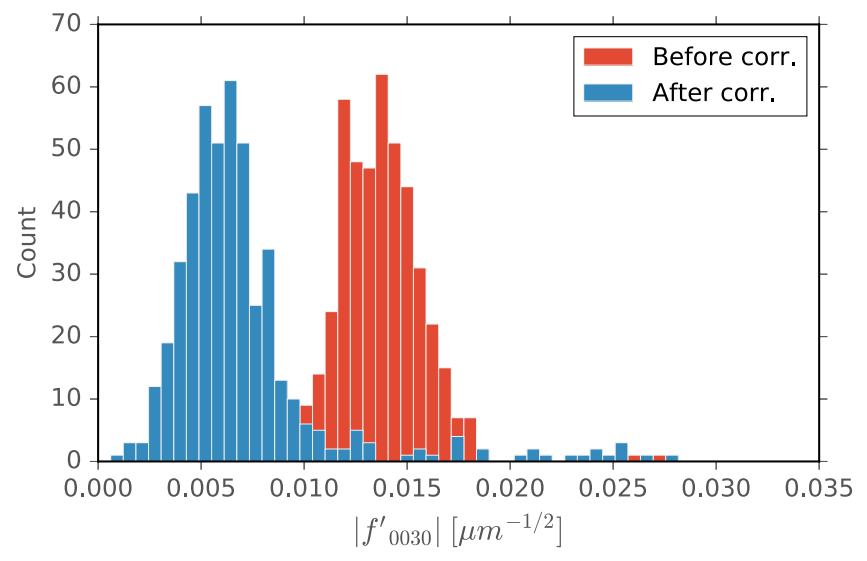

FIG. 24. Histogram of the $\left|f_{0030}^{\prime}\right|$ ac-dipole resonance driving term, related to the $3 Q_{y}$ resonance, measured in LHC BPMs with and without correction of skew sextupole errors in the ATLAS (IR1) insertion.
(CMS), see for example Table III, skew-sextupole compensation also significantly reduced the change to tune separation and working point anticipated during LHC luminosity leveling, which may actually represent the most significant operational benefit arising from the correction.

\section{LINEAR REOPTIMIZATION}

Correction of normal sextupole errors in IR5 (CMS) improved linear optics stability as a function of the applied crossing scheme, as seen in Fig. 16. In spite of this improvement however, there remained a nonzero dependence of beta-beat on crossing angle. Figure 25 (top, center) compares beta-beating measured in Beam 2 after application of the nonlinear corrections and with the operational crossing scheme applied, to that obtained after linear optics commissioning at flat orbit. A slight deterioration of the beta-beat is observed. Similar results were obtained for Beam 2. Figure 25 (bottom) also shows significant normalized dispersion beating generated upon application of the crossing scheme. The degradation of

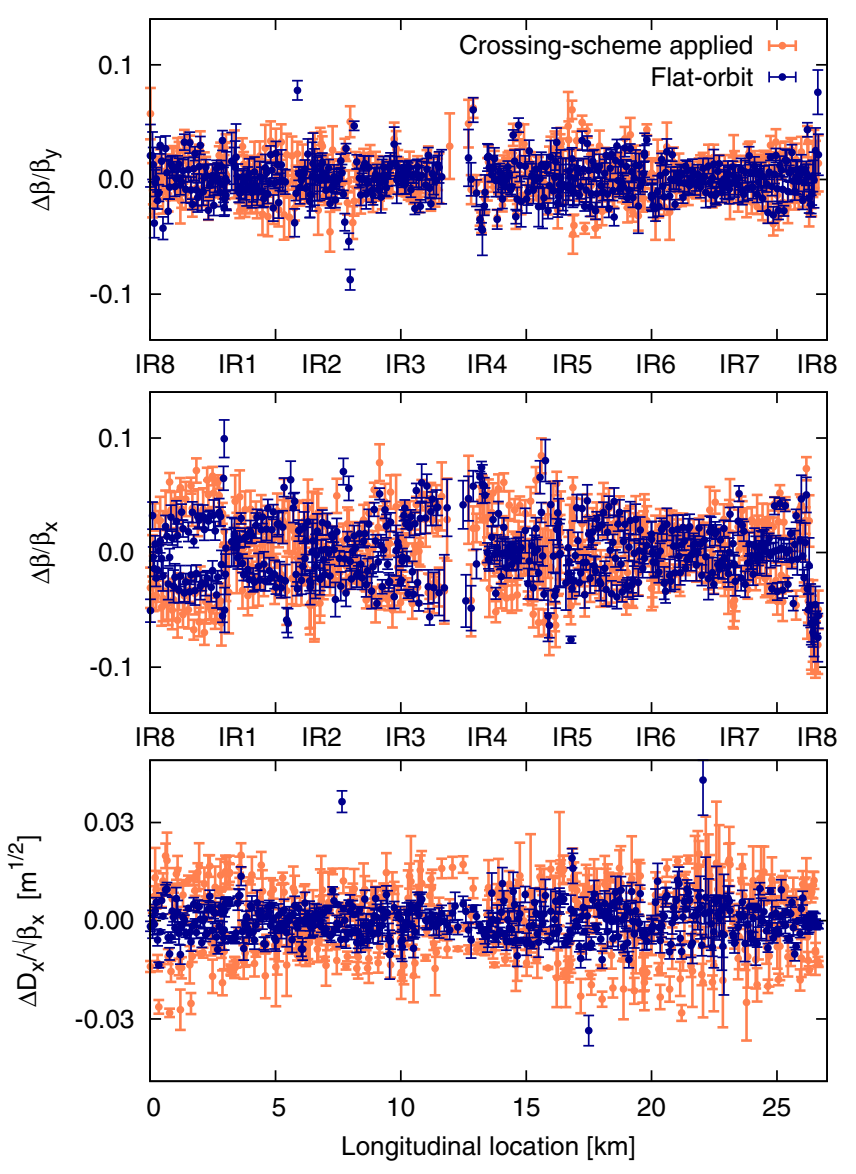

FIG. 25. Beta-beating (top,center) and normalized dispersion (bottom) of LHC Beam 2, measured at flat orbit after application of global linear optics corrections, and at the operational crossing scheme after application of all nonlinear corrections in 2017. 

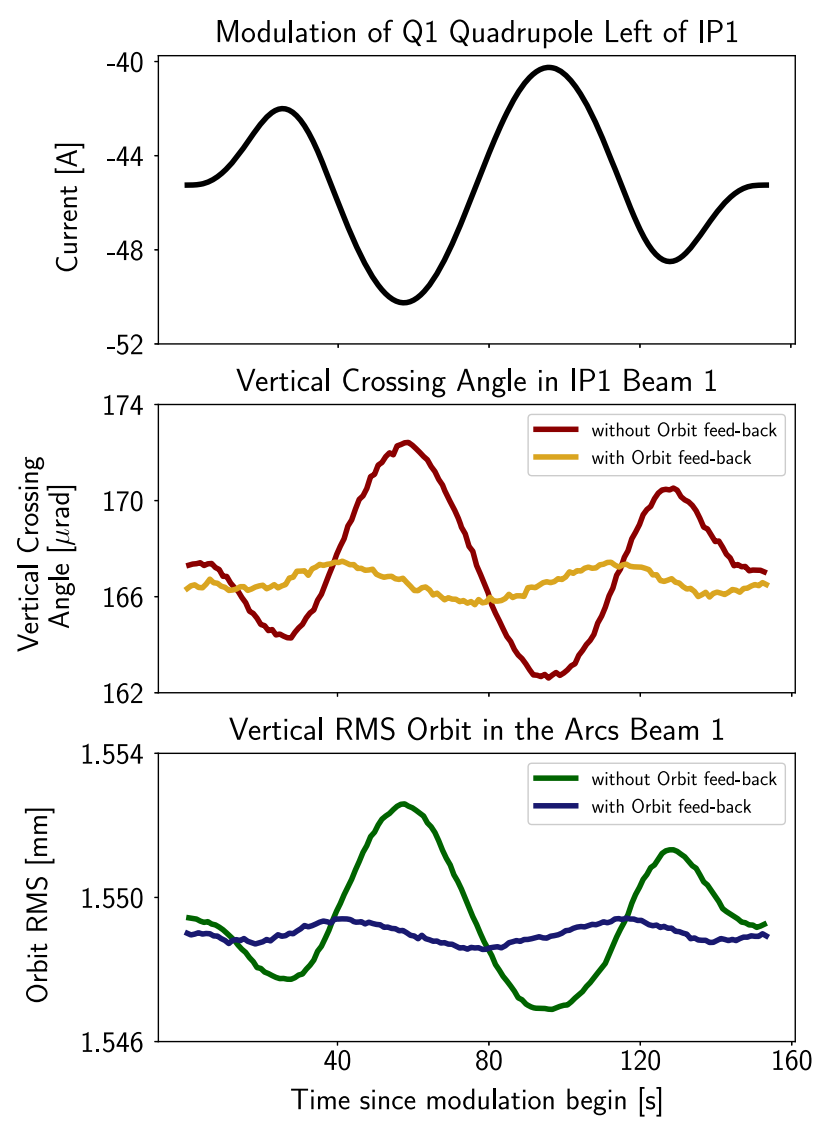

FIG. 26. Orbit leakage during K-modulation measurements performed in the operational configuration of the $\beta^{*}=0.3 \mathrm{~m}$.

$\beta$-beat and normalized dispersion upon application of the crossing scheme prompted a linear reoptimization in the operational configuration. This is in contrast to previous years where optics correction was only performed at flat orbit.

With the operational crossing scheme applied optics measurement via acv-dipole excitation could be performed as normal (see Sec. II and [7]). K-modulation of the triplet quadrupoles however, generated significant closed-orbit distortion when attempted with the crossing angles applied. This is seen in Fig. 26, which shows clear modulation of the RMS orbit in the arcs (Fig. 26 bottom, green), as well as changes to the measured crossing angle (Fig. 26 center, red). K-modulation requires precise measurement of tune shift from quadrupole modulation, at the level of $\Delta Q_{x, y} \leq 1 \times 10^{-5}$. Such large orbit modulations can distort the $\beta$-measurement due to feed-down from nonlinear magnets or errors in the arcs and from any residual nonlinear errors in the low- $\beta$ IRs. To limit distortion of the tune modulation by feed-down, K-modulation was performed, for the first time, with an active orbit feedback (OFB) operational during the measurement. As seen in Fig. 26 (yellow and blue) application of the OFB significantly improved orbit
TABLE VII. Distortion of $\beta^{*}$ and $\beta^{*}$-waist shift measurements in IR1 (ATLAS) due to orbit leakage during K-modulation. Measurements are for the operational configuration of the $\beta^{*}=$ $0.3 \mathrm{~m}$ optics. Values quoted are the relative change in $\beta^{*}$, and the shift to $\beta^{*}$-waist, between $\mathrm{K}$-modulation measurements performed with and without the orbit feedback active.

\begin{tabular}{lcc}
\hline \hline & Beam 1 & Beam 2 \\
\hline$\Delta \beta_{x}^{*}[\%]$ & $1.3 \pm 0.8$ & $-0.2 \pm 0.3$ \\
$\Delta \beta_{y}^{*}[\%]$ & $4 \pm 2$ & $-2.3 \pm 0.4$ \\
$\beta_{x}^{*}$-waist shift [cm] & $0.36 \pm 0.5$ & $-5.2 \pm 0.7$ \\
$\beta_{y}^{*}$-waist shift [cm] & $-2 \pm 1$ & $-2.7 \pm 0.6$ \\
\hline \hline
\end{tabular}

leakage during the measurement. Table VII details the change in $\beta^{*}$ and $\beta^{*}$-waist location inferred from $\mathrm{K}$ modulation of the $\beta^{*}=0.3 \mathrm{~m}$ optics in IR1 (ATLAS), when the K-modulation measurements were performed in the operational configuration with and without the orbit feedback applied. Orbit leakage during this K-modulation measurement distorted the inferred $\beta^{*}$ by up to several percent, and the waist location by several centimeters, relative to measurements performed with the orbit feedback applied. Such errors on the $\beta^{*}$ and waist shift are unacceptable for LHC operation. Introduction of an orbit feedback into K-modulation measurement in 2017 was therefore essential to facilitate measurement of $\beta^{*}$ with IR crossing angle orbit bumps applied. This then allowed for the first correction of the linear optics in the true operational configuration of the LHC. An example of the linear reoptimization can be seen in Fig. 27 which compares the vertical $\beta$-beat of Beam 1 before and after application of the linear reoptimization in the operational configuration.

Table VIII summarizes the RMS $\beta$-beat and normalized dispersion obtained at various stages in the commissioning process. In the operational configuration (with nonlinear corrections applied) some non-negligible deterioration to RMS $\beta$-beat is observed relative to flat orbit in 2017 (highlighted in bold in Table VIII). Application of linear optics corrections in the operational

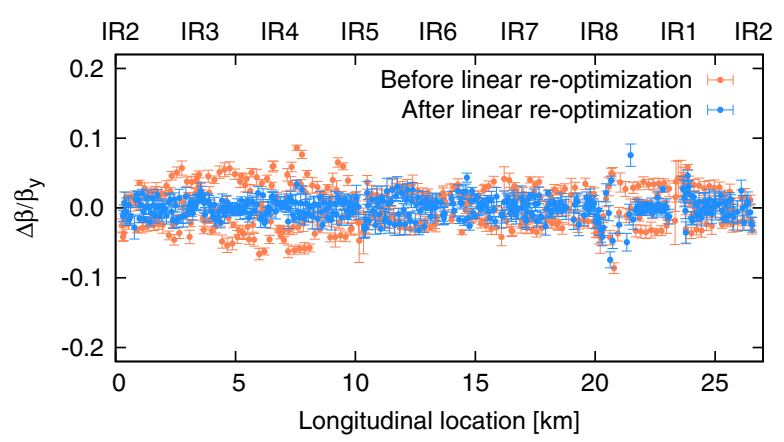

FIG. 27. Vertical $\beta$-beat of LHC Beam 1 before and after reoptimization of linear optics corrections at the operational configuration of the crossing scheme. 
TABLE VIII. Comparison of linear optics quality at various stages throughout the 2017 commissioning of the LHC Achromatic Telescope Squeeze (ATS) optics at $0.4 \mathrm{~m}$.

\begin{tabular}{lccc}
\hline \hline & $\begin{array}{c}\text { 2017 Flat-orbit } \\
\text { (linear corrs) }\end{array}$ & $\begin{array}{c}\text { 2017 OP-crossing } \\
\text { (after NL corrs) }\end{array}$ & $\begin{array}{c}\text { 2017 OP-crossing } \\
\text { (after linear reoptimization) }\end{array}$ \\
\hline Beam 1 $\left.\beta_{x}\right|_{\text {RMS }}[\%]$ & 2.3 & 2.7 & 2.5 \\
Beam 1 $\left.\beta_{y}\right|_{\text {RMS }}[\%]$ & 1.5 & $\mathbf{2 . 8}$ & 1.3 \\
Beam 2 $\left.\beta_{x}\right|_{\text {RMS }}[\%]$ & 2.6 & $\mathbf{3 . 2}$ & 2.5 \\
Beam $\left.2 \beta_{y}\right|_{\text {RMS }}[\%]$ & 1.5 & 1.8 & 1.8 \\
Beam 1 $\left.\frac{\Delta D_{x}}{\sqrt{\beta_{x}}}\right|_{\text {RMS }}\left[10^{-2} \mathrm{~m}^{-\frac{1}{2}}\right]$ & 0.45 & 0.96 & 0.73 \\
Beam 2 $\left.\frac{\Delta D_{x}}{\sqrt{\beta_{x}}}\right|_{\text {RMS }}\left[10^{-2} \mathrm{~m}^{-\frac{1}{2}}\right]$ & 0.58 & 1.11 & 0.61 \\
\hline \hline
\end{tabular}

configuration however, restored the RMS $\Delta \beta / \beta$ of the most severely affected planes to the same level achieved at flat orbit (highlighted in italic in Table VIII). This is also illustrated in Figs. 28 and 29, which show the final beta-beat obtained for the operational configuration of the LHC at $\beta^{*}=0.4 \mathrm{~m}$ in 2017 (blue), compared to that obtained at flat orbit (black) after the linear optics commissioning described in Sec. II.

As seen in Table VIII, application of the crossing scheme approximately doubled the normalized dispersion beating. Reiteration of the linear optics in the operational configuration reduced the dispersion beating of Beam 2 to the level obtained for flat orbit. The RMS $\Delta D_{x} / \sqrt{\beta_{x}}$ of Beam 1 was also partially reduced, but remained worse than that obtained at flat orbit. Measurement quality for Beam 1 however was particularly low, with typical BPM uncertainties larger than the calculated RMS. Figure 30 compares
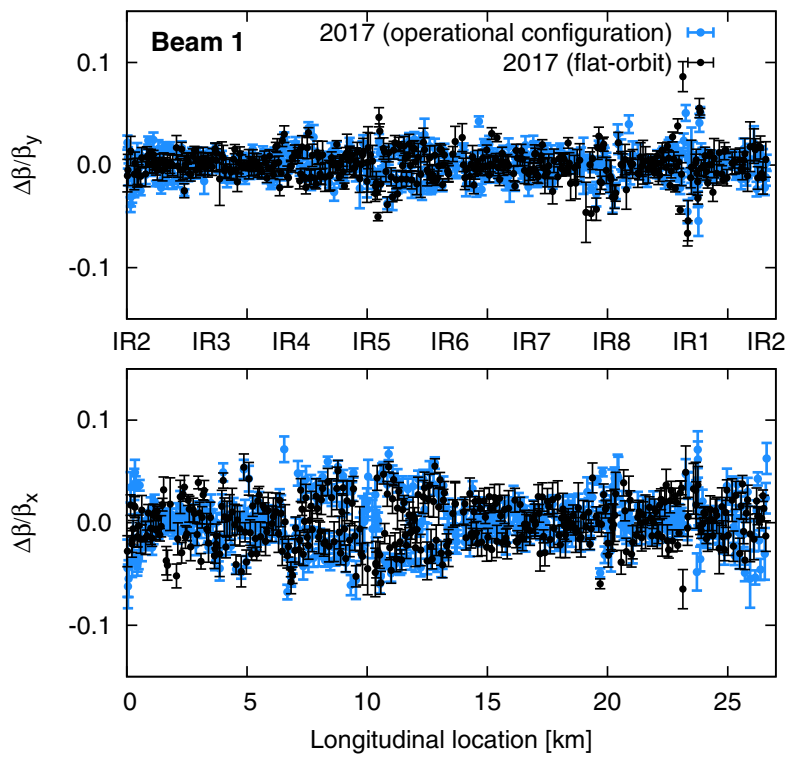

FIG. 28. $\beta$-beat in Beam 1 measured for flat orbit and the operational configuration at $\beta^{*}=0.4 \mathrm{~m}$ in 2017 . the final normalized dispersion beat obtained for the operational configuration at $\beta^{*}=0.4 \mathrm{~m}$ in 2017 to that obtained with a flat orbit.

While control of beta-beat and normalized dispersion globally around the ring is of significant concern, the key parameter of relevance to luminosity production is $\beta^{*}$. Table IX shows $\beta^{*}$ values measured via K-modulation in the ATLAS (IR1) and CMS (IR5) insertions for the operational configuration in 2017 and compares them to the values obtained at flat orbit for the LHC optics in 2016. Note that the LHC also transitioned from its nominal design optics scheme to Achromatic Telescope Squeeze (ATS) optics in 2017 [38,40]. Comparing measurements before and after linear reoptimization, the new optics correction procedure systematically reduced $\beta^{*}$ errors in the operational configuration. For all planes control of $\beta^{*}$ was achieved at the level of $1 \%-2 \%$ for the operational

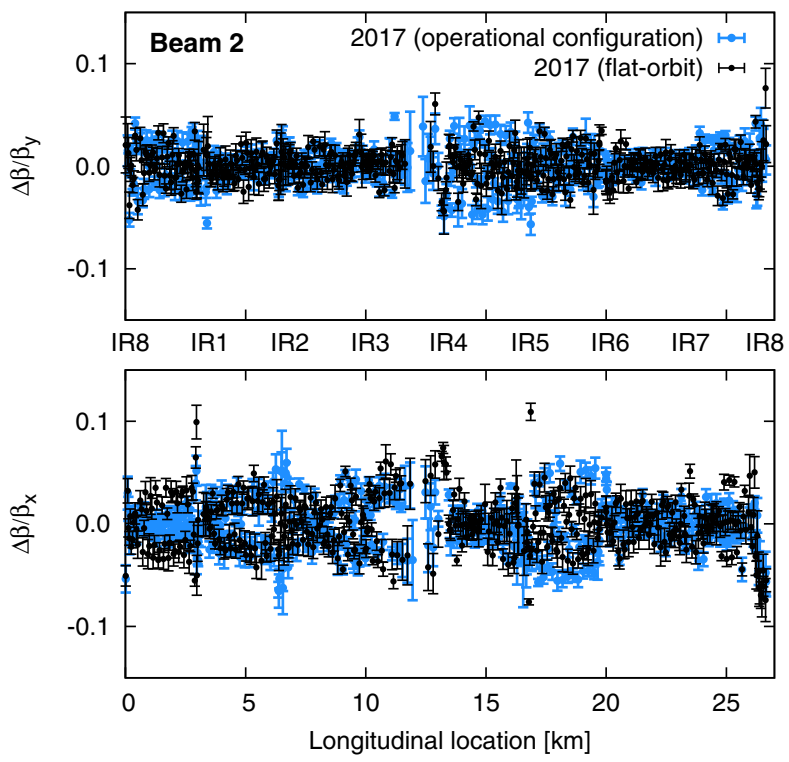

FIG. 29. $\beta$-beat in Beam 2 measured for at flat orbit and the operational configuration at $\beta^{*}=0.4 \mathrm{~m}$ in 2017 . 

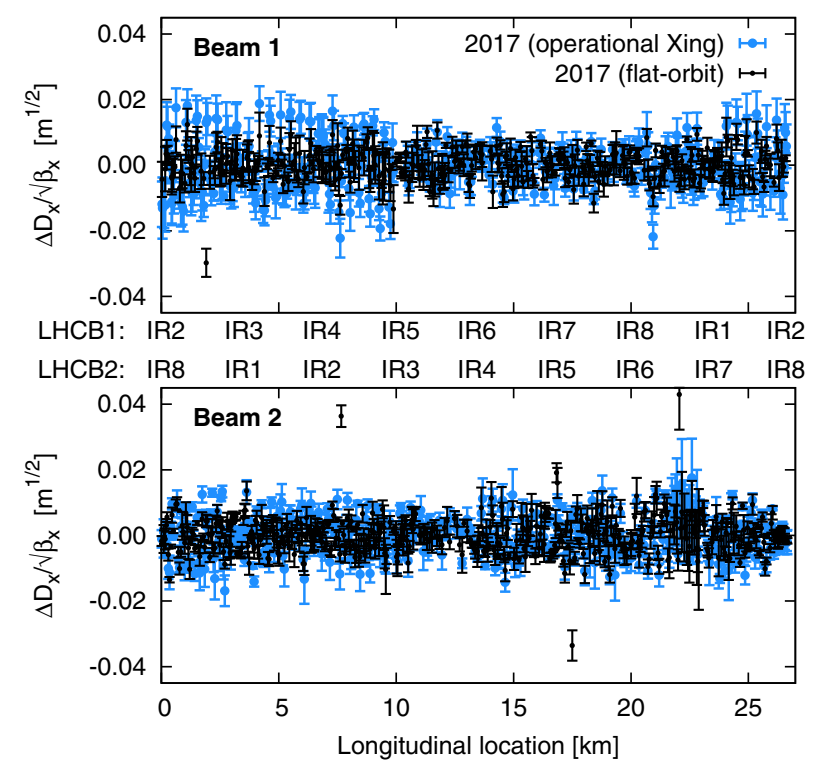

FIG. 30. Normalized dispersion at flat orbit for Beam 1 (top) and Beam 2 (bottom), measured for flat orbit and for the operational configuration at $\beta^{*}=0.4 \mathrm{~m}$ in 2017 .

configuration, a level which was only obtained at flat orbit in previous years of LHC operation.

The $\beta^{*}$-imbalance in Table IX can be related to an expected luminosity imbalance between the ATLAS (IR1) and CMS (IR5) insertions. Prior to linear reoptimization of the optics the predicted luminosity imbalance due to $\beta^{*}$ discrepancies would have been

$$
\frac{L_{\mathrm{CMS}}}{L_{\mathrm{ATLAS}}}=0.974 \pm 0.004
$$

after linear reoptimization this value is significantly improved:

$$
\frac{L_{\mathrm{CMS}}}{L_{\mathrm{ATLAS}}}=1.003 \pm 0.004
$$

TABLE X. $\quad \beta^{*}$ obtained for the operational configuration of the IR crossing scheme for $\beta^{*}=0.3 \mathrm{~m}$ ATS optics in 2017 .

\begin{tabular}{lcc}
\hline \hline & \multicolumn{2}{c}{2017 ATS (OP-crossing) } \\
\cline { 2 - 3 } & Beam 1 & Beam 2 \\
\hline$\left.\beta_{x}\right|_{\text {RMS }}$ & $2.0 \%$ & $1.8 \%$ \\
$\left.\beta_{y}\right|_{\text {RMS }}$ & $1.8 \%$ & $1.7 \%$ \\
$\left.\beta_{x}^{*}\right|_{\text {IP1 }}[\mathrm{cm}]$ & $30.7 \pm 0.2$ & $30.0 \pm 0.3$ \\
$\left.\beta_{y}^{*}\right|_{\text {IP1 }}[\mathrm{cm}]$ & $30.5 \pm 0.2$ & $30.0 \pm 0.1$ \\
$\left.\beta_{x}^{*}\right|_{\text {IP5 }}[\mathrm{cm}]$ & $30.7 \pm 0.2$ & $29.8 \pm 0.1$ \\
$\left.\beta_{y}^{*}\right|_{\text {IP5 }}[\mathrm{cm}]$ & $30.1 \pm 0.1$ & $30.4 \pm 0.2$ \\
Luminosity imbalance & \multicolumn{2}{c}{$0.2 \% \pm 0.6 \%$} \\
\hline \hline
\end{tabular}

The change in LHC optics commissioning strategy implemented in 2017, to consider optics quality with the operational configuration of the crossing scheme, therefore represents a significant gain in regard to operation of the collider.

Commissioning of the LHC optics performed prior to 2017 exclusively focused on the linear optics at flat orbit. From results presented in Sec. III and IV, it can be surmised that nonlinear errors and the crossing scheme represent a contribution to the beta-beat at the level of $\sim 1-2 \%$ at $\beta^{*}=0.4 \mathrm{~m}$. This contribution to the linear optics errors was neither considered nor corrected in previous years, thus earlier results relating to linear optics control at low- $\beta^{*}$ in the LHC [7] may actually represent a slight overestimate of the optics quality achieved during actual operation of the collider for luminosity production. Compensation of the linear optics in the true operational configuration now represents a standard component of LHC commissioning strategy. Table X, for example, shows the beta-beat obtained for the operational crossing scheme at $\beta^{*}=0.3 \mathrm{~m}$, which has also been used for operation in 2017 and 2018. With additional iteration of the optics corrections in the operational configuration, the RMS $\beta$-beat was further improved compared to $\beta^{*}=0.4 \mathrm{~m}$ commissioning, while control of $\beta^{*}$ remains around the $1 \%-2 \%$ level.

TABLE IX. $\quad \beta^{*}$ obtained for the operational configuration of the IR crossing scheme for $\beta^{*}=0.4 \mathrm{~m}$ ATS optics in 2017 (before and after linear reoptimization), contrasted to the optics quality obtained for the nominal LHC optics at

\begin{tabular}{|c|c|c|c|c|c|c|}
\hline & \multicolumn{2}{|c|}{2017 ATS before } & \multicolumn{2}{|c|}{2017 ATS after } & \multicolumn{2}{|c|}{2016 nominal } \\
\hline & Beam $1[\mathrm{~cm}]$ & Beam 2 [cm] & Beam 1 [cm] & Beam $2[\mathrm{~cm}]$ & Beam $1[\mathrm{~cm}]$ & Beam $2[\mathrm{~cm}]$ \\
\hline$\left.\beta_{x}^{*}\right|_{\mathrm{IP} 1}$ & $40.4 \pm 0.1$ & $39.0 \pm 0.1$ & $39.9 \pm 0.1$ & $39.9 \pm 0.1$ & $39.8 \pm 0.5$ & $39.8 \pm 0.1$ \\
\hline$\left.\beta_{y}^{*}\right|_{\mathrm{IP} 1}$ & $38.5 \pm 0.1$ & $41.3 \pm 0.2$ & $40.8 \pm 0.4$ & $40.1 \pm 0.1$ & $40.1 \pm 0.1$ & $40.1 \pm 0.1$ \\
\hline$\left.\beta_{x}^{*}\right|_{\text {IP5 }}$ & $40.6 \pm 0.1$ & $40.5 \pm 0.2$ & $40.3 \pm 0.2$ & $40.2 \pm 0.1$ & $39.9 \pm 0.2$ & $39.5 \pm 0.1$ \\
\hline$\left.\underline{\beta_{y}^{*}}\right|_{\text {IP5 }}$ & $40.3 \pm 0.1$ & $41.6 \pm 0.3$ & $40.2 \pm 0.2$ & $39.6 \pm 0.1$ & $40.1 \pm 0.1$ & $39.6 \pm 0.2$ \\
\hline
\end{tabular}
flat orbit in 2016. 


\section{CONCLUSIONS}

Prior to 2017, the low- $\beta^{*}$ optics commissioning strategy for the LHC was exclusively concerned with linear optics. Since 2017 this procedure has undergone a major revision, extending the strategy to encompass also nonlinear magnetic errors in low- $\beta^{*}$ insertions, and to account for perturbation of the linear optics by feed-down from such nonlinear errors when crossing angle orbit bumps are applied in the experimental IRs. Commissioning strategy has in essence moved from a purely linear approach to a combined linear and nonlinear commissioning. This alternative strategy continued to be deployed and refined during 2018 operation, and combined linear/ nonlinear commissioning is foreseen to become a mainstay of LHC operation for the remainder of its life.

Where previously no nonlinear corrections were applied in the low- $\beta^{*}$ insertions of the LHC, beam-based corrections have now been implemented for normal and skew octupole, and normal and skew sextupole errors, yielding a range of operational benefits. Normal octupole correction eliminated radical distortions of the tune footprint through the $\beta^{*}$ squeeze, providing a stable baseline upon which to implement Landau damping of instabilities. The normal octupole correction also markedly improved the performance of beam instrumentation, specifically the continuous measurement of tune and coupling via the LHC BBQ system. This was essential in order to obtain the highquality K-modulation data necessary to correct $\beta^{*}$ and luminosity imbalance at end-of-squeeze. Correction of the sextupoles and skew octupoles also significantly improved the stability of tune separation and linear coupling as a function of crossing angle. Since Landau damping in the LHC is critically dependent on the magnitude of the linear coupling compared to the tune separation, the nonlinear corrections played a significant role in facilitating the introduction of crossing angle leveling into LHC operation since 2017.

Where results from linear optics commissioning have previously been reported, only the optics measured with a flat closed-orbit (an orbit with the operational crossing angle bumps in the experimental insertions removed) was considered. A non-negligible change to the $\beta$-beat was observed however, as function of the crossing angle. Arising from feed-down of nonlinear errors in the low$\beta^{*}$ IRs, this represents an additional source of linear optics imperfections present in the true operational configuration, which had not been considered earlier in LHC operation. To obtain the high-quality optics control desired in the LHC, it is therefore necessary to also consider the role nonlinear sources in the experimental IRs play in perturbing the linear optics.

Correction of normal sextupole errors in the CMS insertion helped improve the stability of the linear optics as a function of the crossing scheme. After all nonlinear corrections were applied however, a non-negligible deterioration to the full operational configuration still remained (relative to the optics quality obtained during initial commissioning at flat orbit). The linear commissioning strategy was therefore extended, to include a reoptimization of $\beta^{*}$; global $\beta$-beat; and normalized dispersion, with the operational crossing scheme applied (and after application of the nonlinear corrections). This was the first time linear optics measurements at the LHC were performed with the crossing angle orbit bumps applied. An update to the $\mathrm{K}$-modulation method was required, to measure with an orbit feed-back operational, in order to minimize distortion of the tune modulation by feed-down from nonlinear magnets in the arcs and from any residual nonlinearities in the IRs. After reoptimization, a linear optics quality was achieved which is comparable to that obtained at flat orbit and in previous years.

Correction of nonlinear errors in low- $\beta^{*}$ insertions is a question of longstanding interest to various current and historical colliders, as well as an emerging challenge for new machines such as the HL-LHC, FCC, and SuperKEK. Beam-based correction of multiple species of nonlinear errors in the low- $\beta^{*}$ IRs, and for the influence of these nonlinearities on the linear optics, has been achieved for the first time in the LHC using a variety of different linear and nonlinear observables. The corrections have been applied in regular operation with clear benefit to the machine. Of particular relevance to future colliders, such as the HL-LHC and FCC, the IR-nonlinear correction significantly improved the lifetime of the LHC beams during dedicated tests at very small $\beta^{*}=0.14 \mathrm{~m}$. This is well below the $\beta^{*}$ values currently used in LHC operation $\left(\beta^{*}=0.40-0.25 \mathrm{~m}\right)$ but is of relevance to HL-LHC operation $\left(\beta^{*}=0.15 \mathrm{~m}\right)$. Several key resonance driving terms, corresponding to the $4 Q_{x}$ and $3 Q_{y}$ resonances, were also reduced upon application of the relevant multipole corrections. The revised LHC commissioning strategy, based on a combined commissioning of the linear and nonlinear optics, represents a new approach which will be applied in future LHC operations and provides an initial template for commissioning of the HL-LHC in the coming years.

\section{ACKNOWLEDGMENTS}

Profuse thanks go to the CERN operations group and LHC Engineers In Charge for the extensive support lent to the commissioning and machine development studies which contributed to the results presented here. Similar thanks go to the LHC collimation team for helping facilitate the large amplitude beam excitations necessary for the nonlinear commissioning, and to the Beam Instrumentation group for their continual support of all the optics studies done on the LHC. Finally many thanks go to the magnet field quality (FiDeL) team at CERN for many productive discussions regarding the field quality in the LHC insertions. 
[1] R. Tomás, O. Bruning, M. Giovannozzi, P. Hagen, M. Lamont, F. Schmidt, G. Vanbavinckhove, M. Aiba, R. Calaga, and R. Miyamoto, CERN Large Hadron Collider optics model, measurements, and corrections, Phys. Rev. ST Accel. Beams 13, 121004 (2010).

[2] G. Vanbavinckhove, Optics measurements and corrections for colliders and other storage rings, Ph.D. thesis, Universiteit van Amsterdam, 2012, https://cds.cern.ch/record/ 1533084 ? 1 .

[3] A. Langner and R. Tomás, Optics measurement algorithms and error analysis for the proton energy frontier, Phys. Rev. ST Accel. Beams 18, 031002 (2015).

[4] R. Tomás, T. Bach, J. Coello, V. Kain, M. Kuhn, A. S. Langner, Y. I. Levinsen, K. S. B Li, E. H. Maclean, V. Maier, N. Magnin, M. J. McAteer, T. H. B. Persson, P. K. Skowronski, R. Westenberger, and S. White, Prospects for the LHC optics measurements and corrections at higher energy, in Proceedings of 5th Int. Particle Accelerator Conf. (IPAC'14), Dresden, Germany (2014), pp. 1046-1048, https://doi.org/10.18429/JACoWIPAC2014-TUPRO018.

[5] M. Aiba, S. Fartoukh, A. Franchi, M. Giovannozzi, V. Kain, M. Lamont, R. Tomás, G. Vanbavinckhove, J. Wenninger, F. Zimmermann, R. Calaga, and A. Morita, First $\beta$-beating measurement and optics analysis for the CERN Large Hadron Collider, Phys. Rev. ST Accel. Beams 12, 081002 (2009).

[6] R. Tomás, R. Calaga, A. Langner, Y. I. Levinsen, E. H. Maclean, T. H. B. Persson, P. K. Skowronski, M. Stzelczyk, G. Vanbavinckhove, and R. Miyamoto, Record low $\beta$-beating in the LHC, Phys. Rev. ST Accel. Beams 15, 091001 (2012).

[7] T. Persson, F. Carlier, J. Coello de Portugal, A. GarciaTabares Valdivieso, A. Langner, E. H. Maclean, L. Malina, P. Skowronski, B. Salvant, R. Tomás, and A. C. Garcia Bonilla, LHC optics commissioning: A journey towards 1\% optics control, Phys. Rev. Accel. Beams 20, 061002 (2017).

[8] L. C. Teng, Error analysis for the low- $\beta$ quadrupoles of the Tevatron collider, Technical Report No. FERMILAB-TM1097, 1982, http://inspirehep.net/record/177853.

[9] J. Wei and M. Harrison, The RHIC project-design, status, challenges, and perspectives, in Multi-GeV highperformance accelerators and related technology. Proceedings, 16th RCNP International Symposium, Osaka, Japan, 1997 (1997), C97-03-12.1, pp. 198-206, www .rhichome.bnl.gov/AP/ap_notes/RHIC_AP_123.ps, FERMILAB-TM-1097.

[10] O. Brüning et al. LHC Design Report v.1: The LHC main ring, CERN, 2004, https://cds.cern.ch/record/782076?ln.

[11] F. Pilat, Y. Luo, N. Malitsky, and V. Ptitsyn, Beam-based non-linear optics corrections in colliders, in Proceedings of 21st Particle Accelerator Conf. (PAC'05), Knoxville, TN, USA, 2005, paper WOAC007, pp. 601-605, http://accelconf.web.cern.ch/AccelConf/p05/PAPERS/ WOAC007.PDF.

[12] W. Fischer, J. Beebe-Wang, Y. Luo, S. Nemesure, and L. Rajulapati, RHIC proton beam lifetime increase with 10- and 12-pole correctors, in Proceedings of the
International Particle Accelerator Conference, Kyoto, Japan (ICR, Kyoto, 2010), THPE099.

[13] G. Apollinari, I. Béjar Alonso, O. Bruning, M. Lamont, and L. Rossi, High-Luminosity Large Hadron Collider (HL-LHC): Preliminary design report, CERN Technical Report No. CERN-2015-005, 2015, https://cds.cern.ch/ record/2116337? In.

[14] M. Giovannozzi, S. Fartoukh, and R. De Maria, Specification of a system of correctors for the triplets and separation dipoles of the LHC upgrade, in Proceedings of the 4th International Particle Accelerator Conference, IPAC-2013, Shanghai, China, 2013 (JACoW, Shanghai, China, 2013), WEPEA048, http://accelconf.web.cern.ch/ AccelConf/IPAC2013/papers/wepea048.pdf.

[15] M. Giovannozzi, Field quality and DA, in 6th $H L-L H C$ Collaboration Meeting, Paris, France, 2016, Espace St Martin, https://indico.cern.ch/event/549979/contributions/ 2263210/.

[16] F. Carlier, J. Coello, S. Fartoukh, E. Fol, A. GarcíaTabares, M. Giovannozzi, M. Hofer, A. Langer, E. H. Maclean, L. Malina, L. Medina, T. H. B. Persson, P. Skowronski, R. Tomás, F. Van der Veken, and A. Wegscheider, Optics measurement and correction challenges for the HL-LHC, CERN Technical Report No. CERNACC-2017-0088, 2017, https://cds.cern.ch/record/2290899.

[17] H. Sugimoto, SuperKEKB, Presentation at CERN-ICFA Workshop on Advanced Optics Control, 2015, https:// indico.cern.ch/event/349643/overview.

[18] Daniel Schulte, Optics challenges for future hadron colliders, Presentation at CERN-ICFA Workshop on Advanced Optics Control, 2015, https://indico.cern.ch/ event/349643/overview.

[19] M. Benedikt, D. Schulte, J. Wenninger, and F. Zimmerman, Challenges for highest energy circular colliders, CERN Technical Report No. CERN-ACC-2014-0153, 2014, https://cds.cern.ch/record/1742294.

[20] E. Cruz-Alaniz, A. Seryi, E. H. Maclean, R. Martin, and R. Tomás, Non linear field correction effects on the dynamic aperture of the FCC-hh, in Proceedings of IPAC 17, Copenhagen, Denmark, 2017, TUPVA038, http://accelconf.web .cern.ch/AccelConf/ipac2017/papers/tupva038.pdf.

[21] E. H. Maclean, Nonlinear optics commissioning in the LHC, in Proceedings of the 7th Evian Workshop, Evian, France (2016), https://indico.cern.ch/event/578001/contributions/ 2366314/attachments/1374391/2158727/2016_EvianPaper .pdf.

[22] L. R. Carver, M. Schenk, R. de Maria, K. Li, D. Amorim, N. Biancacci, X. Buffat, E. Maclean, E. Metral, K. Lasocha, T. Lefevre, T. Levens, and B. Salvant, MD1831: Single bunch instabilities with Q and non-linear errors, CERN Technical Report No. CERN-ACC-NOTE2017-0012, 2017, https://cds.cern.ch/record/2253143?ln.

[23] E. H. Maclean, R. Tomás, F. Schmidt, and T. H. B. Persson, Measurement of nonlinear observables in the Large Hadron Collider using kicked beams, Phys. Rev. ST Accel. Beams 17, 081002 (2014).

[24] S. White, E. Maclean, and R. Tomás, Direct amplitude detuning measurement with ac dipole, Phys. Rev. ST Accel. Beams 16, 071002 (2013). 
[25] E. H. Maclean, Non-linear modelling and machine set-up, Presentation at LHC OMC review, 2013, http://indico.cern .ch/getFile.py/access?contribId=5\&sessionId=0\&resId=1\& materialId=slides\&confId=246159.

[26] E. H. Maclean, F. Carlier, M. Giovannozzi, T. H. B. Persson, and R. Tomás, Effect of linear coupling on nonlinear observables at the LHC, in Proceedings of 8th Int. Particle Accelerator Conf. (IPAC'17), Copenhagen, Denmark, WEPIK092 (2017), pp. 3151-3154, https://doi.org/10.18429/ JACoW-IPAC2017-WEPIK092.

[27] T. Persson, E. H. Maclean, R. Tomás, and Y. I. Levinsen, Non-linear coupling studies in the LHC, in Proceedings of 6th Int. Particle Accelerator Conf. (IPAC'15), Richmond, VA, USA, TUPTY042 (2015), pp. 2105-2107, https://doi .org/10.18429/JACoW-IPAC2015TUPTY042.

[28] R. Tomás, T. H. B. Persson, and E. H. Maclean, Amplitude dependent closest tune approach, Phys. Rev. Accel. Beams 19, 071003 (2016).

[29] T. H. B. Persson, M. Gasior, E. H. Maclean, O. Jakub, R. Tomás, D. Valuch, D. A. Wierichs, and F. S. Carlier, Suppression of Amplitude dependent closest tune approach and first tests of the ADT as an AC-dipole (MD 1412), Technical Report No. CERN-ACC-Note-2016-0057, 2016, https://cds.cern.ch/record/2220704?ln.

[30] E. H. Maclean, T. H. B. Persson, and R. Tomás, Amplitude dependent closest tune approach generated by normal and skew octupoles, in Proceedings of 8th Int. Particle Accelerator Conf. (IPAC'17), Copenhagen, Denmark, WEPIK091 (2017), pp. 3147-3150, https://doi.org/10.18429/ JACoW-IPAC2017-WEPIK091.

[31] T. Persson, R. Tomás, and E. H. Maclean, Phys. Rev. Accel. Beams 22, 051001 (2019).

[32] L. R. Carver, D. Amorim, N. Biancacci, X. Buffat, K. S. B. Li, E. Métral, B. Salvant, and M. Schenk, Destabilising effect of linear coupling in the LHC, in Proceedings of 8th Int. Particle Accelerator Conf. (IPAC'17), Copenhagen, Denmark, THPAB040 (2017), pp. 3791-3794, https://doi .org/10.18429/JACoW-IPAC2017-THPAB040.

[33] L. R. Carver, X. Buffat, K. Li, E. Metrel, and M. Schenk, Transverse beam instabilities in the presence of linear coupling in the Large Hadron Collider, Phys. Rev. Accel. Beams 21, 044401 (2018).

[34] E. H. Maclean, R. Tomás, F. S. Carlier, M. S Camillocci, J. Coello de Portugal, E. Fol, K. Fuchsberger, A. GarciaTabares Valdivieso, M. Giovannozzi, M. Hofer, L. Malina, T. H. B. Persson, P. K. Skowronski, and A. Wegscheider, Detailed review of the LHC optics commissioning for the nonlinear era, CERN Technical Report No. CERN-ACC2019-0029, 2019, http://cds.cern.ch/record/2655741?ln.

[35] R. Calaga, R. Tomás, and F. Zimmerman, BPM calibration independent LHC optics correction, CERN Technical Report No. CERN-LHC-PROJECT-Report-1039, 2007, http://cds.cern.ch/record/1058520?ln.

[36] R. Tomás, O. Bruning, S. Fartoukh, M. Giovannozzi, Y. Papaphilippou, F. Zimmermann, R. Calaga, S. Peggs, and A. Franchi, Procedures and accuracy estimates for betabeat correction in the LHC, in Proceedings of the 10th European Particle Accelerator Conference, Edinburgh,
Scotland, 2006 (EPS-AG, Edinburgh, Scotland, 2006), WEPCH047.

[37] R. Tomás, M. Aiba, A. Franchi, and U. Iriso, Review of linear optics measurement and correction for charged particle accelerators, Phys. Rev. Accel. Beams 20, 054801 (2017).

[38] S. Fartoukh, Towards the LHC Upgrade using the LHC well-characterized technology, CERN Technical Report No. CERN-sLHC-PROJECT-Report-0049, 2012, http:// cds.cern.ch/record/1301180?ln.

[39] S. Fartoukh, Achromatic telescopic squeezing scheme and application to the LHC and its luminosity upgrade, Phys. Rev. ST Accel. Beams 16, 111002 (2013).

[40] S. Fartoukh, R. Bruce, F. Carlier, J. Coello De Portugal, A. Garcia-Tabares, E. Maclean, L. Malina, A. Mereghetti, D. Mirarchi, T. Persson, M. Pojer, L. Ponce, S. Redaelli, B. Salvachua, P. Skowronski, M. Solfaroli, R. Tomás, D. Valuch, A. Wegscheider, and J. Wenninger, Experimental validation of the Achromatic Telescopic Squeezing (ATS) scheme at the LHC, J. Phys. Conf. Ser. 874, 012010 (2017).

[41] A. Franchi, Studies and measurements of linear coupling and nonlinearities in hadron circular accelerators, Ph.D. thesis, Universität Frankfurt, 2006, http://publikationen.ub .uni-frankfurt.de/frontdoor/index/index/docId/2270.

[42] R. Calaga, R. Tomás, and A. Franchi, Betatron coupling: Merging Hamiltonian and matrix approaches, Phys. Rev. ST Accel. Beams 8, 034001 (2005).

[43] T. Persson and R. Tomás, Improved control of the betatron coupling in the Large Hadron Collider, Phys. Rev. ST Accel. Beams 17, 051004 (2014).

[44] T. Persson et al., Transverse coupling measurements with high intensity beams using driven oscillations, in Proceedings of 9th Int. Particle Accelerator Conf. (IPAC'18), Vancouver, Canada, MOPMF047 (2018), pp. 208-211, https://doi.org/10.18429/JACoW-IPAC2018-MOPMF047.

[45] T. H. B. Persson, Y. I. Levinsen, R. Tomás, andE. H. Maclean, Chromatic coupling correction in the Large Hadron Collider, Phys. Rev. ST Accel. Beams 16, 081003 (2013).

[46] CERN FiDeL group documentation on the magnetic model of the LHC, Technical Report, https://hc-div-mms.web .cern.ch/lhc-div-mms/tests/MAG/Fidel/.

[47] O. Bruning, S. Fartoukh, M. Giovannozzi, and T. Risselada, Dynamic aperture studies for the LHC separation dipoles, LHC Technical Report No. LHC Project Note 349, 2004, https://cds.cern.ch/record/742967?ln.

[48] R. Tomás, M. Giovannozzi, and R. de Maria, Nonlinear correction schemes for the phase 1 LHC insertion region upgrade and dynamic aperture studies, Phys. Rev. ST Accel. Beams 12, 011002 (2009).

[49] E. H. Maclean, R. Tomás, M. Giovannozzi, and T. H. B. Persson, First measurement and correction of nonlinear errors in the experimental insertions of the CERN Large Hadron Collider, Phys. Rev. ST Accel. Beams 18, 121002 (2015).

[50] R. Tomás, Adiabaticity of the ramping process of an ac dipole, Phys. Rev. ST Accel. Beams 8, 024401 (2005).

[51] S. Mönig, E. H. Maclean, T. H. B. Persson, J. M. Coello de Portugal, A. Langner, and R. Tomás, Short term dynamic aperture with AC dipoles, in Proceedings of 7th Int. Particle Accelerator Conf. (IPAC'16), Busan, 
Korea, THPMR044 (2016), pp. 3496-3499, https://doi .org/10.18429/JACoW-IPAC2016-THPMR044.

[52] F. S. Carlier, R. Tomás, E. H. Maclean, and T. H. B. Persson, First experimental demonstration of dynamic aperture measurements with LHC AC dipoles, Phys. Rev. Accel. Beams 22, 031002 (2019).

[53] E. H. Maclean, F. S. Carlier, J. M. Coello de Portugal, A. Garcia-Tabares, M. Giovannozzi, L. Malina, T. H. B Persson, P. K. Skowronski, and R. Tomás, New methods for measurement of nonlinear errors in LHC experimental IRs and their application in the HL-LHC, in Proceedings of 8th Int. Particle Accelerator Conf. (IPAC'17), Copenhagen, Denmark, WEPIK093 (2017), pp. 3155-3158, https://doi.org/10.18429/JACoW-IPAC2017-WEPIK093.

[54] R. Tomás, Normal form of particle motion under the influence of an ac dipole, Phys. Rev. ST Accel. Beams 5, 054001 (2002).

[55] F. Carlier, R. Tomás, E. H. Maclean, and T. H. B. Persson, Nonlinear correction strategies for the LHC using resonance driving terms, in Proceedings of 9th Int. Particle Accelerator Conf. (IPAC'18), Vancouver, Canada, MOPFM032 (2018), pp. 161-164, https://doi.org/10 .18429/JACoW-IPAC2018-MOPMF032.

[56] F. Carlier, R. Tomás, and E. H. Maclean, Measurement and Correction of Resonance Driving Terms in the LHC (to be published).

[57] MAD-Methodical Accelerator Design, http://mad.web .cern.ch/mad/.

[58] É. Forest, F. Schmidt, and E. McIntosh, Introduction to the polymorphic tracking code, CERN Technical Report No. CERN-SL-2002-044 (AP), 2002, http://cds.cern.ch/ record/573082/files/sl-2002-044.pdf.

[59] J. W. Dilly, E. H. Maclean, F. Carlier, M. Hofer, and R. Tomás, Report from LHC MD 3311: Amplitude detuning at end-of-squeeze, Technical Report, 2018 (to be published).

[60] A. Boccardi, M. Gasior, R. Jones, and R. J. Steinhagen, An overview of the LHC transverse diagnostics systems, LHC Technical Report No. LHC Project Report 1166, 2009, http://cds.cern.ch/record/1156346?ln.

[61] M. Gasior and R. Jones, The principle and first results of betatron tune measurement by direct diode detection, LHC Technical Report No. LHC-Project-Report 853, 2005, http://cds.cern.ch/record/883298?ln.

[62] E. H. Maclean, M. Giovannozzi, T. H. B. Persson, R. Tomás, and J. Wenninger, Understanding the tune, coupling, and chromaticity dependence of the LHC on Landau octupole powering, CERN Technical Report No. CERNATS-Note-2013-023 TECH, 2013, https://cds.cern.ch/ record/1541981? ln.

[63] E. H. Maclean, M. Giovannozzi, W. Herr, Y. I. Levinsen, G. Papotti, T. H. B. Persson, P. K. Skowronski, R. Tomás, and J. Wenninger, Understanding the tune, coupling, and chromaticity dependence of the LHC on Landau octupole powering, in Proceedings of the 4th International Particle Accelerator Conference, IPAC2013, Shanghai, China, 2013 (JACoW, Shanghai, China, 2013), TUPWO048.

[64] D. A. Wierichs, Uncertainties and noise in coupling measurements at the Large Hadron Collider, CERN internal report, Report No. CERN-STUDENTS-Note-2016-168, 2016, http://cds.cern.ch/record/2212256.

[65] E.H. Maclean, New optics correction approaches in 2017, Proceedings of the 8th Evian Workshop (Evian, 2017), https://indico.cern.ch/event/663598/contributions/ 2781846/attachments/1573573/2752298/2017_evian.pdf.

[66] F. S. Carlier, J. M. Coello de Portugal, J. W. Dilly, E. Fol, A. Garcia-Tabares Valdivieso, E. H. Maclean, L. Malina, T. H. B. Persson, P. K. Skowronski, and R. Tomás, MD2723-Amplitude detuning studies at $6.5 \mathrm{TeV}$ with various configurations of the crossing scheme, CERN Technical Report No. CERN-ACC-NOTE-2018-0022, 2018, http://cds.cern.ch/record/2306325.

[67] S. Fartoukh et al., ATS MDs in 2016, CERN Technical Report No. CERN-ACC-2017-0003, 2017, https://cds.cern .ch/record/2242513?ln.

[68] E. H. Maclean, F. Carlier, M. S Camillocci, K. Fuchsberger, M. Giovannozzi, T. H. B. Persson, and R. Tomás, Report from LHC MDs 1391 and 1483: Tests of new methods for study of nonlinear errors in the LHC experimental insertions, CERN Technical Report No. CERNACC-NOTE-2018-0035, 2017, http://cds.cern.ch/record/ 2314410.

[69] G. Guignard, Betatron coupling and related impact of radiation, Phys. Rev. E 51, 6104 (1995).

[70] R. Tomás, Optimizing the global coupling knobs for the LHC, CERN Technical Report No. CERN-ATS-Note-2012019 MD, 2012, http://cds.cern.ch/record/1422434?ln.

[71] E. H. Maclean, R. Tomás, T. H. B. Persson, and F. S. Carlier, Report from LHC MD 2171: Amplitude dependent closest tune approach from normal and skew octupoles, CERN Technical Report No. CERN-ACC-Note-20180027, 2018, http://cds.cern.ch/record/2310163. 